\title{
Deciphering Multi-way Interactions in the Human Genome
}

\author{
Stephen Lindsly ${ }^{1, \dagger}$, Can Chen ${ }^{2,3, \dagger}$, Sam Dilworth ${ }^{4}$, Sivakumar Jeyarajan ${ }^{1}$, Walter Meixner ${ }^{1}$, Cooper \\ Stansbury $^{1}$, Anthony Cicalo ${ }^{1}$, Nicholas Beckloff ${ }^{5}$, Charles Ryan ${ }^{6,7}$, Amit Surana ${ }^{8}$, Max Wicha ${ }^{9}$, Lindsey \\ A. Muir ${ }^{1}$, and Indika Rajapakse ${ }^{1,2, *}$ \\ ${ }^{1}$ Department of Computational Medicine and Bioinformatics, University of Michigan, Ann Arbor, MI 48109 USA \\ ${ }^{2}$ Department of Mathematics, University of Michigan, Ann Arbor, MI 48109 USA \\ ${ }^{3}$ Department of Electrical Engineering and Computer Science, University of Michigan, Ann Arbor 48109 USA \\ ${ }^{4}$ iReprogram, Ann Arbor, MI 48105 USA \\ ${ }^{5}$ Oxford Nanopore Technologies, Oxford, OX4 4DQ UK \\ ${ }^{6}$ Medical Scientist Training Program, University of Michigan, Ann Arbor, MI 48109 USA \\ ${ }^{7}$ Program in Cellular and Molecular Biology, University of Michigan, Ann Arbor, MI 48109 USA \\ ${ }^{8}$ Raytheon Technologies Research Center, East Hartford, CT 061084 warnings USA \\ ${ }^{9}$ Department of Hematology/Oncology, University of Michigan, Ann Arbor, MI 48109 USA \\ ${ }^{\dagger}$ These authors contributed equally to this work. \\ ${ }^{*}$ To whom correspondence should be addressed (indikar@umich.edu).
}

\begin{abstract}
Chromatin architecture, a key regulator of gene expression, is inferred through chromatin contacts. However, classical analyses of chromosome conformation data do not preserve multi-way relationships. Here we use long sequencing reads to map genome-wide multi-way contacts and investigate higher order chromatin organization of the human genome. We use the theory of hypergraphs for data representation and analysis, and quantify higher order structures in primary human fibroblasts and B lymphocytes. Through integration of multi-way contact data with chromatin accessibility, gene expression, and transcription factor binding data, we introduce a data-driven method to extract transcriptional clusters.
\end{abstract}




\section{Introduction}

Structural features of the genome are integral to regulation of gene expression and corresponding outcomes in cellular phenotype $[1,2,3]$. Genome-wide chromosome conformation capture (Hi-C) enables large scale study of genome architecture by capturing all pairwise interactions (contacts) between genomic loci. Through analysis of pairwise interactions, multiway interactions may be inferred, but not directly captured [4]. Recently, an extension of Hi-C enabled preservation and sequencing of long reads (e.g. Pore-C) [5], which can be used to unambiguously identify sets of contacts among multiple loci. These data will undoubtedly clarify higher order structures in the genome, but new frameworks are required for analysis and representation of the multidimensional data captured in this assay.

We collected Pore-C data from two cell types and constructed hypergraphs to represent the multidimensional relationships of multi-way contacts among loci. Hypergraphs are similar to graphs, but have hyperedges instead of edges, with each hyperedge connecting any number of nodes instead of two nodes $[6,7,8]$. In our hypergraph framework, nodes are genomic loci, and hyperedges are multi-way contacts among loci. Incidence matrices are used to represent hypergraphs, where matrix rows represent genomic loci and columns are individual hyperedges. This representation enables quantitative measurements of genome organization through hypergraph entropy and the comparison of different cell types through hypergraph distance. In addition, we integrate Pore-C data with multiple other data modalities to identify biologically relevant multi-way interactions.

We use the following definitions. Entropy: a measure of structural order in the genome. Hyperedge: an extension of edges where each edge can contain any number of nodes. Hypergraph: an extension of graphs containing multiple hyperedges. Incidence matrix: a representation for hypergraphs where rows are nodes and columns are hyperedges. Transcription cluster: a group of genomic loci that colocalize for efficient gene transcription.

\section{Results}

\subsection{Capturing Multi-way Contacts}

We conducted Pore-C experiments using adult human dermal fibroblasts and obtained additional publicly available Pore-C data from B lymphocytes [5]. The experimental protocol for Pore-C is similar to Hi-C, including cross-linking, restriction digestion, and ligation of adjacent ends followed by sequencing (Figure 1A). Alignment of Pore-C long reads to the genome enables fragment identification and construction of multi-way contacts (Figure 1B).

Hypergraphs represent multi-way contacts, where individual hyperedges contain at least two loci (Figure 1C, left). Hypergraphs provide a simple and concise way to depict multi-way contacts and allow for abstract representations of genome structure. Computationally, we represent multi-way contacts as incidence matrices (Figure 1C, right). For Hi-C data, adjacency matrices are useful for assembly of pairwise genomic contacts. However, since rows and columns represent individual loci, adjacency matrices cannot be used for multi-way contacts in Pore-C data. In contrast, incidence matrices permit more than two loci per contact and provide a clear visualization of multi-way contacts. Multi-way contacts can also be decomposed into pairwise contacts, similar to Hi-C, by extracting all pairwise combinations of loci (Figure 1D). 


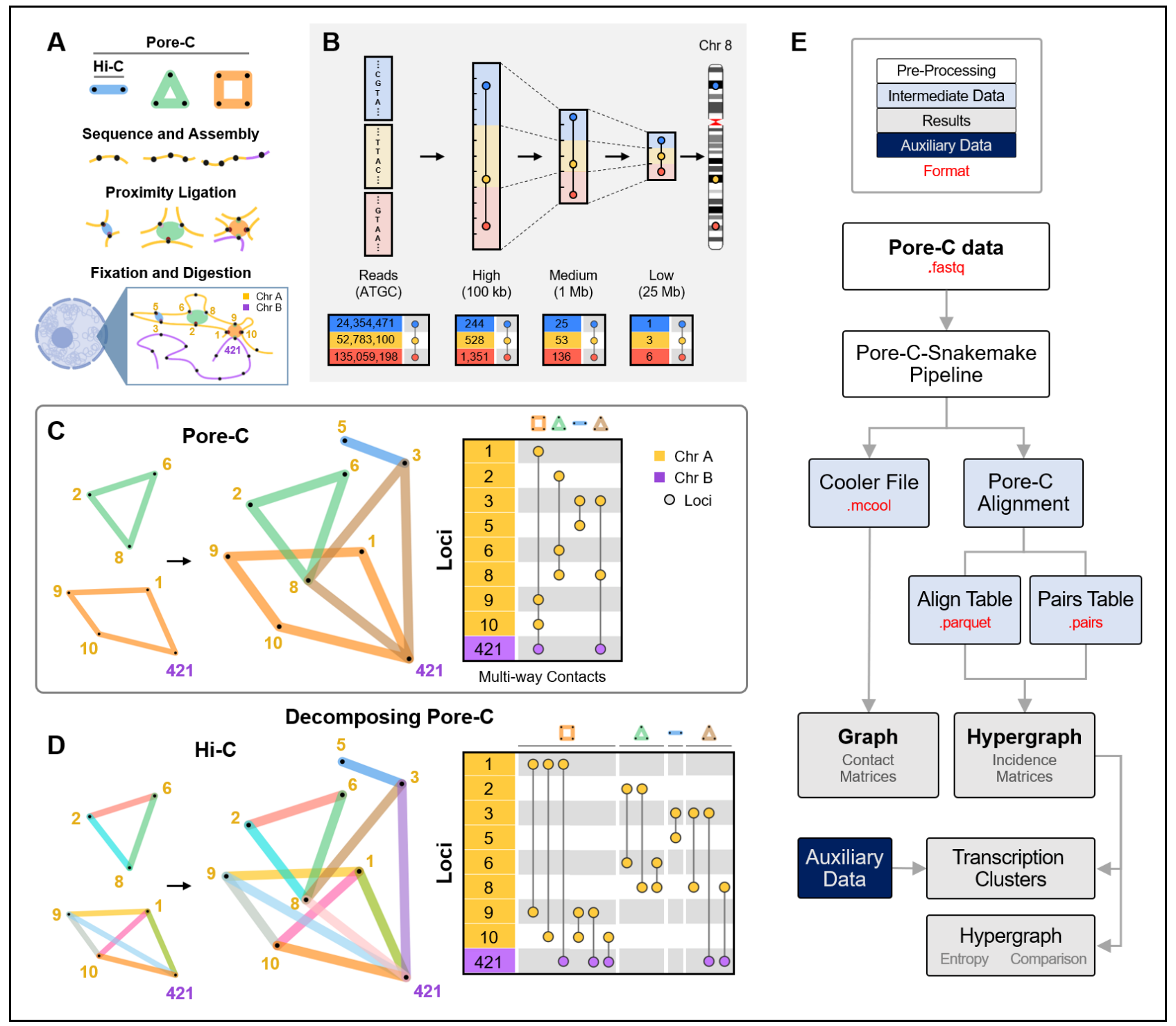

Figure 1: Pore-C experimental and data workflow. (A) The Pore-C experimental protocol, which captures pairwise and multiway contacts (Materials and Methods). (B) Representation of multi-way contacts at different resolutions (top). Incidence matrix visualizations of a representative example from Chromosome 8 in human fibroblasts at each resolution (bottom). The numbers in the left columns represent the location of each genomic locus present in a multi-way contact, where values are either the chromosome base-pair position (read-level) or the bin into which the locus was placed (binning at $100 \mathrm{~kb}, 1$ $\mathrm{Mb}$, or $25 \mathrm{Mb}$ ). (C) Hypergraph representation of Pore-C contacts (left) and an incidence matrix (right) of four multi-way contacts within (yellow-to-yellow) and between (yellow-to-purple) chromosomes. Contacts correspond to examples from A. The numbers in the left column represent a bin in which a locus resides. Each vertical line represents a multi-way contact, with nodes at participating genomic loci. (D) Multi-way contacts can be decomposed into pairwise contacts. Decomposed multi-way contacts can be represented using graphs (left) or incidence matrices (right). Contacts correspond to examples from A and C. (E) Flowchart overview of the computational framework. File type format descriptions are in Table S1.

\subsection{Decomposing Multi-way Contacts}

From our Pore-C experiments using adult human dermal fibroblasts, and additional publicly available Pore-C data from B lymphocytes, we constructed hypergraphs as multiple resolutions (read level, $100 \mathrm{~kb}, 1 \mathrm{Mb}$, and $25 \mathrm{Mb}$ ) [5]. We first analyzed individual chromosomes at $100 \mathrm{~kb}$ resolution by decomposing multi-way contacts into their pairwise contacts. Decomposing Pore-C data into pairwise contacts provides more information than $\mathrm{Hi}-\mathrm{C}$, as each Pore-C read can contain many pairwise contacts [5]. It also allows us to identify topologically associated domains (TADs) using established methods (Materials and Methods, $[9,10,11])$. We demonstrate identification of TAD boundaries from decomposed multi-way contacts and show intraand inter-TAD relationships using multi-way contacts (Figures 2, S1). The loci that frequently participate in these multi-way contacts give rise to the block-like pattern of chromatin interactions often shown in Hi-C data. 


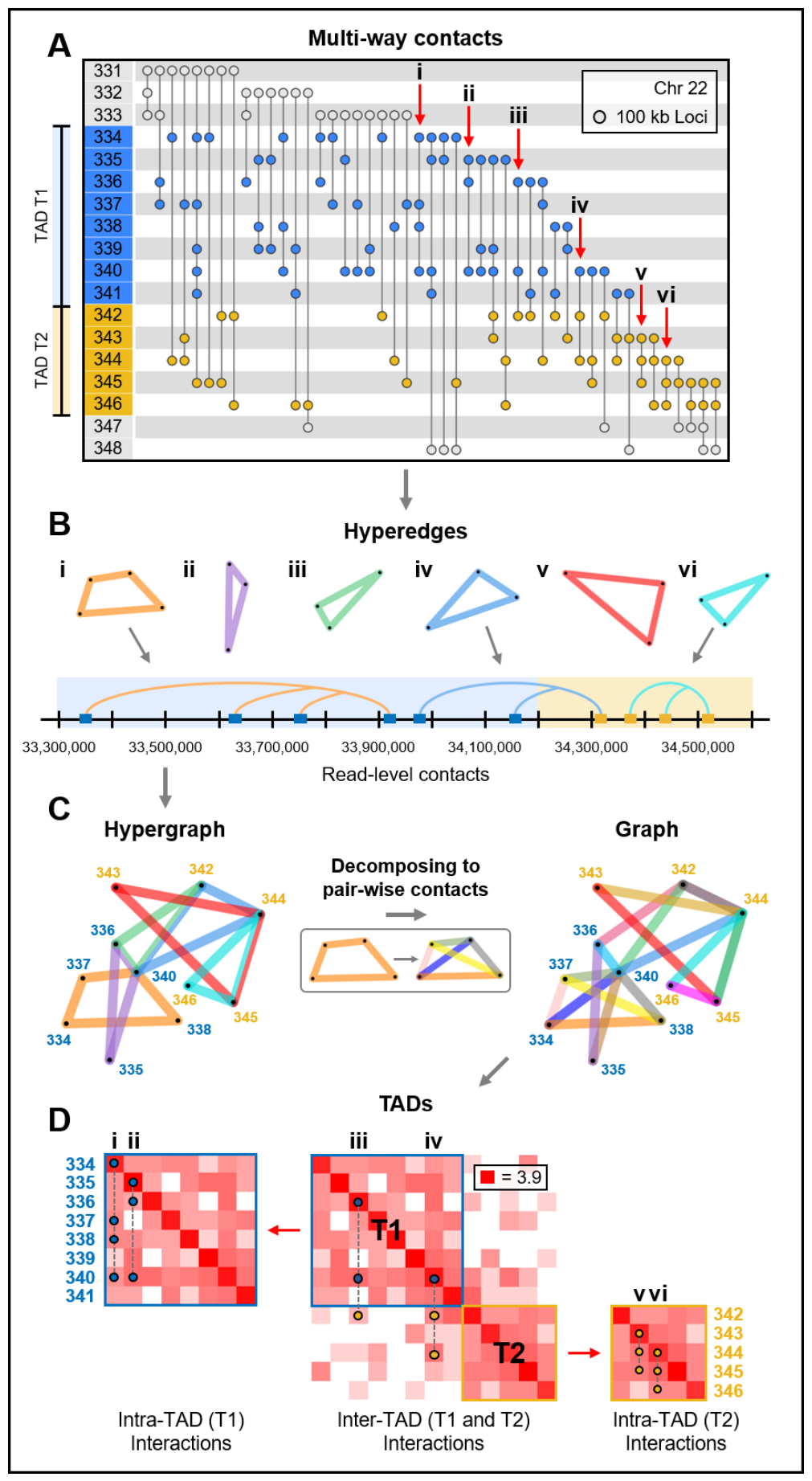

Figure 2: Local organization of the genome. (A) Incidence matrix visualization of a region in Chromosome 22 from fibroblasts (V1-V4). The numbers in the left column represent genomic loci in $100 \mathrm{~kb}$ resolution, vertical lines represent multi-way contacts, where nodes indicate the corresponding locus' participation in this contact. The blue and yellow regions represent two TADs, T1 and T2. The six contacts, denoted by the labels i-vi, are used as examples to show intra- and inter-TAD contacts in B, C, and D. (B) Hyperedge and read-level visualizations of the multi-way contacts i-vi from the incidence matrix in A. Blue and yellow shaded areas (bottom) indicate which TAD each locus corresponds to. (C) A hypergraph is constructed using the hyperedges from B (multiway contacts i-vi from A). The hypergraph is decomposed into its pairwise contacts in order to be represented as a graph. (D) Contact frequency matrices were constructed by separating all multi-way contacts within this region of Chromosome 22 into their pairwise combinations. TADs were computed from the pairwise contacts using the methods from [11]. Example multi-way contacts i-vi are superimposed onto the contact frequency matrices. Multi-way contacts in this figure were determined in $100 \mathrm{~kb}$ resolution after noise reduction, originally derived from read-level multi-way contacts (Materials and Methods). 


\subsection{Chromosomes as Hypergraphs}

To gain a better understanding of genome structure with multi-way contacts, we constructed hypergraphs for entire chromosomes in $1 \mathrm{Mb}$ resolution. We show an incidence matrix of Chromosome 22 as an example in Figure 3A, and in Figure 3B, we visualize Chromosomes 22's distribution of $1 \mathrm{Mb}$ contacts at multiple orders (2-way contacts, 3-way contacts, etc). Figure $3 \mathrm{C}$ highlights the most common intra-chromosome contacts within Chromosome 22 using multi-way contact "motifs", which we use as a simplified way to show hyperedges. Figure 3D shows how multi-way contacts at lower resolutions (25 Mb, $1 \mathrm{Mb})$ are composed of many multi-way contacts at higher resolutions (100 kb, read level), and Figure 3E visualizes the multi-way contacts contained in Figure 3D as a hypergraph.

We also identified multi-way contacts that contain loci from multiple chromosomes. These inter-chromosomal multi-way contacts can be seen in $1 \mathrm{Mb}$ resolution in Figure $3 \mathrm{~F}$ and in $25 \mathrm{Mb}$ resolution for both fibroblasts and B lymphocytes in Figure 4A and 4B, respectively. Figure 4 gives a summary of the entire genome's multi-way contacts, by showing the most common intra- and inter-chromosomal multi-way contacts across all chromosomes. We highlight examples of multi-way contacts with loci that are contained within a single chromosome ("intra only"), spread across unique chromosomes ("inter only"), and a mix of both within and between chromosomes ("intra and inter"). We note that many of the "inter only" contacts observed in Figure 4 may also have intra-chromosomal contacts when viewed at a higher resolution, similar to Figure 3D. Finally, we found the most common inter-chromosomal multi-way contacts across all chromosomes, which we summarize with five example chromosomes in Figure 5 using multi-way contact motifs. These multi-way contacts between distant genomic loci may offer insights into the higher-order structural patterning of the genome and its relationship with transcriptional regulation.

A

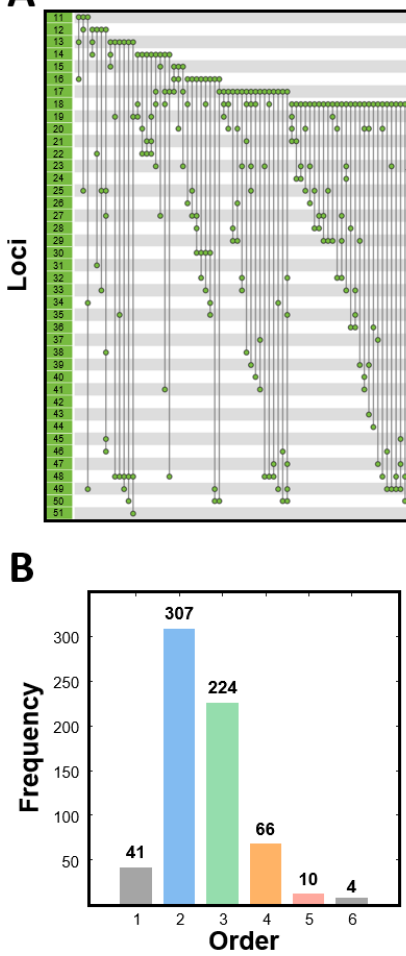

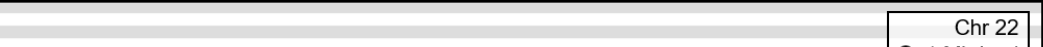
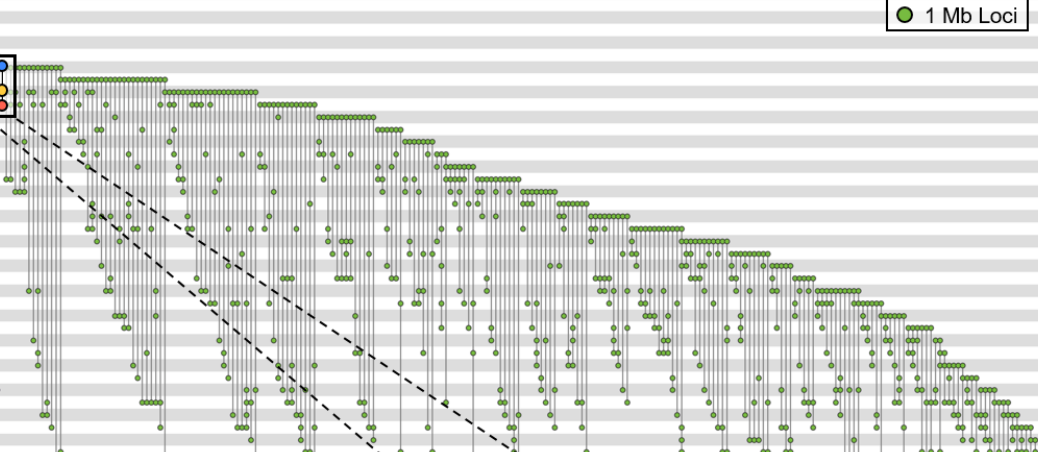

C
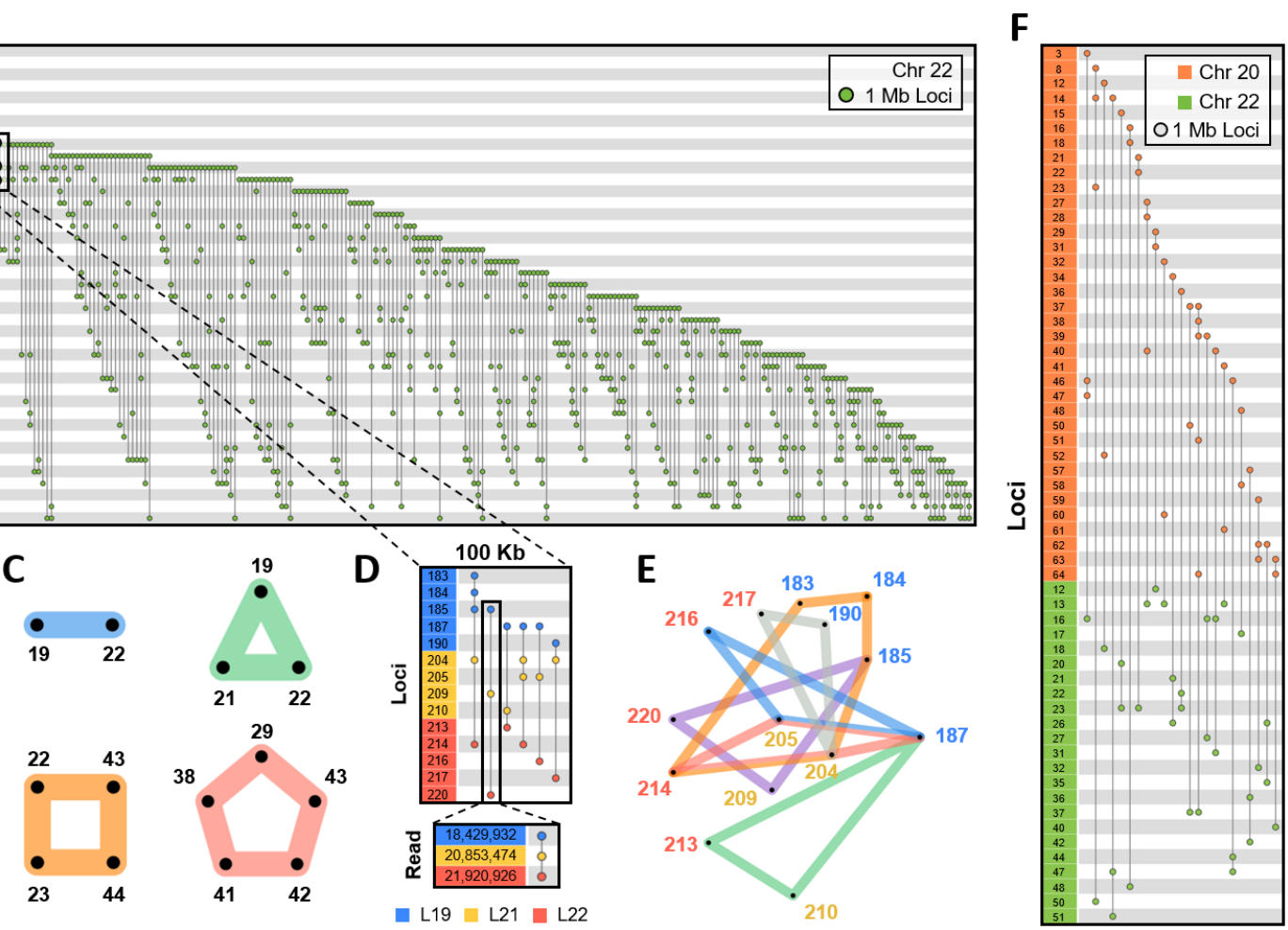

Figure 3: Patterning of intra- and inter-chromosomal contacts. (A) Incidence matrix visualization of Chromosome 22 in fibroblasts. The numbers in the left column represent genomic loci in $1 \mathrm{Mb}$ resolution. Each vertical line represents a multiway contact, in which the nodes indicate the corresponding locus' participation in this contact. (B) Frequencies of Pore-C contacts in Chromosome 22. Bars are colored according to the order of contact. Blue, green, orange, and red correspond to 2-way, 3-way, 4-way, and 5-way contacts. (C) The most common 2-way, 3-way, 4-way, and 5-way intra-chromosome contacts within Chromosome 22 are represented as motifs, color-coded similarly to B. (D) Zoomed in incidence matrix visualization in $100 \mathrm{~kb}$ resolution shows the multi-way contacts between three $1 \mathrm{Mb}$ loci L19 (blue), L21 (yellow), and L22 (red). An example $100 \mathrm{~kb}$ resolution multi-way contact is zoomed to read-level resolution. (E) Hypergraph representation of the 100 kb multi-way contacts from D. Blue, yellow, and red labels correspond to loci L19, L21, and L22, respectively. (F) Incidence matrix visualization of the inter-chromosomal multi-way contacts between Chromosome 20 (orange) and Chromosome 22 (green) in $1 \mathrm{Mb}$ resolution. Within this figure, all data are from one fibroblast sequencing run (V2) and multi-way contacts were determined after noise reduction at $1 \mathrm{Mb}$ or $100 \mathrm{~kb}$ resolution accordingly (Materials and Methods). 


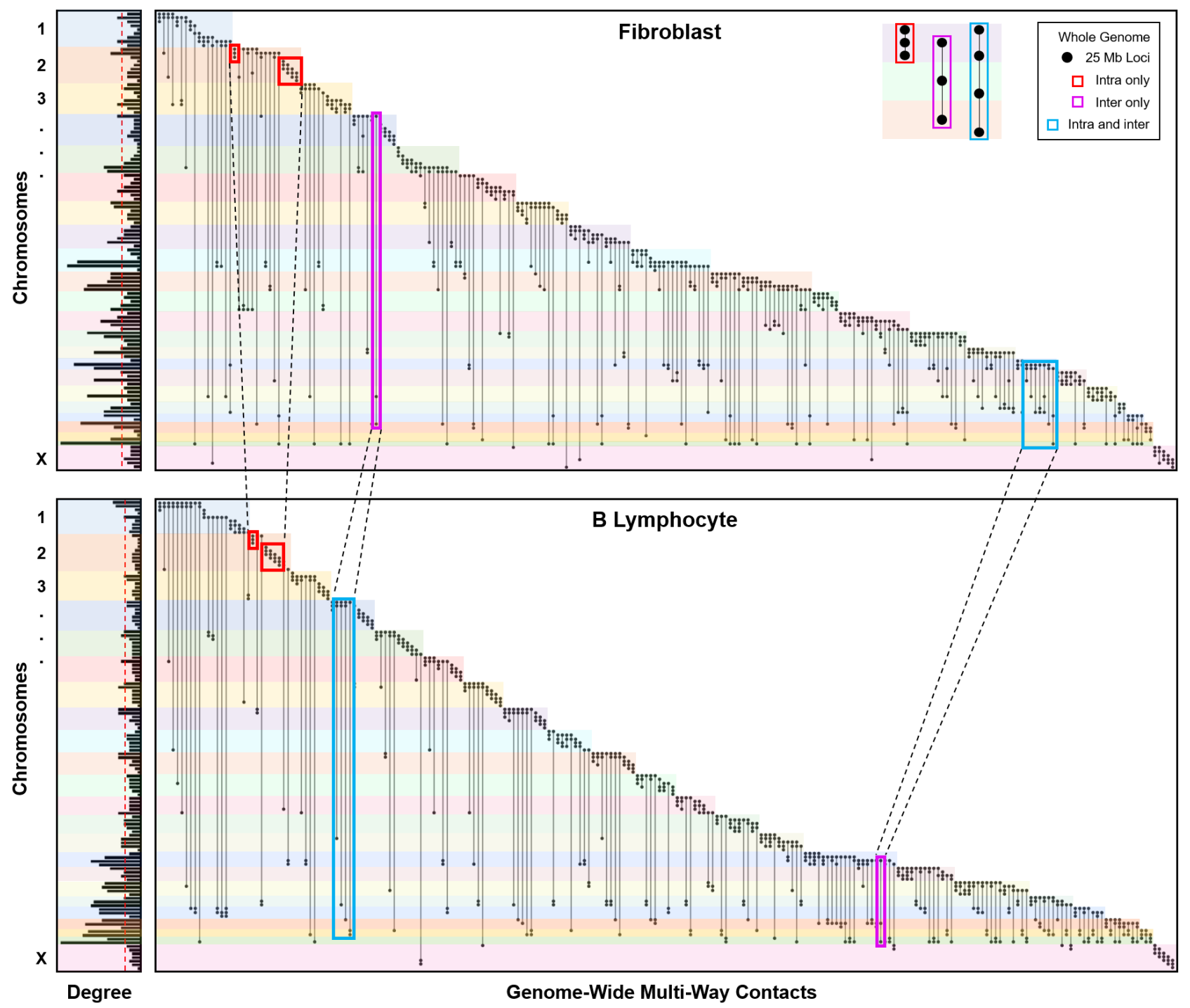

Figure 4: Genome-wide patterning of multi-way contacts. Incidence matrix visualization of the top 10 most common multiway contacts per chromosome. Matrices are constructed at $25 \mathrm{Mb}$ resolution for both fibroblasts (top, V1-V4) and B lymphocytes (bottom). Specifically, 5 intra-chromosomal and 5 inter-chromosomal multi-way contacts were identified for each chromosome with no repeated contacts. If 5 unique intra-chromosomal multi-way contacts are not possible in a chromosome, they are supplemented with additional inter-chromosomal contacts. Vertical lines represent multi-way contacts, nodes indicate the corresponding locus' participation in a multi-way contact, and color-coded rows delineate chromosomes. Highlighted boxes indicate example intra-chromosomal contacts (red), inter-chromosomal contacts (magenta), and combinations of intra- and inter-chromosomal contacts (blue). Examples for each type of contact are shown in the top right corner. Multi-way contacts of specific regions are compared between cell types by connecting highlighted boxes with black dashed lines, emphasizing similarities and differences between fibroblasts and B lymphocytes. Normalized degree of loci participating in the top 10 most common multi-way contacts for each chromosome in fibroblast and B-lymphocytes are shown on the left. Red dashed lines indicate the mean degree for fibroblasts and B lymphocytes (top and bottom, respectively). Genomic loci that do not participate in the top 10 most common multi-way contacts for fibroblasts or B lymphocytes were removed from their respective incidence and degree plots. Multi-way contacts were determined in $25 \mathrm{Mb}$ resolution after noise reduction (Materials and Methods). 


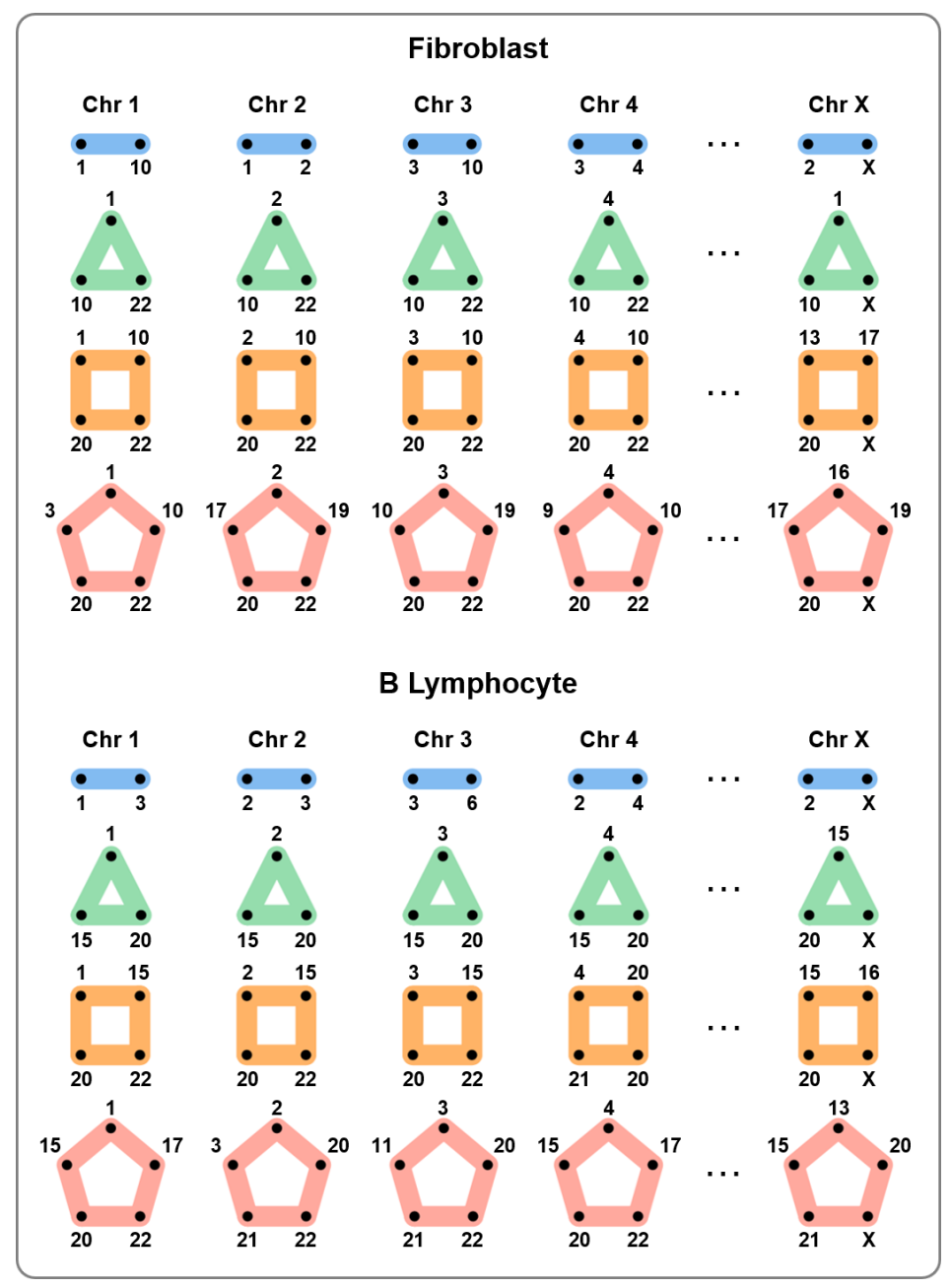

Figure 5: Inter-chromosomal interactions. The most common 2-way, 3-way, 4-way, and 5-way inter-chromosome combinations for each chromosome are represented using motifs from fibroblasts (top, V1-V4) and B lymphocytes (bottom). Rows represent the combinations of 2-way, 3-way, 4-way, and 5-way inter-chromosome interactions, and columns are the chromosomes. Interchromosomal combinations are determined using $25 \mathrm{Mb}$ resolution multi-way contacts after noise reduction (Materials and Methods) and are normalized by chromosome length. Here we only consider unique chromosome instances (i.e. multiple loci in a single chromosome are ignored).

\subsection{Transcription Clusters}

Genes are transcribed in short sporadic bursts and transcription occurs in localized areas with high concentrations of transcriptional machinery $[12,13,14]$. This includes transcriptionally engaged polymerase and the accumulation of specialized proteins, called transcription factors. Multiple genomic loci can colocalize at these areas for more efficient transcription. In fact, it has been shown using fluorescence in situ hybridization (FISH) that genes frequently colocalize during transcription [15]. Simulations have also provided evidence that genomic loci which are bound by common transcription factors can self-assemble into clusters, forming structural patterns commonly observed in Hi-C data [14]. We refer to these instances of highly concentrated areas of transcription machinery and genomic loci as transcription clusters. The colocalization of multiple genomic loci in transcription clusters naturally leads to multi-way contacts, but these interactions cannot be fully captured from the pairwise contacts of Hi-C. Multi-way contacts derived from Pore-C reads can detect interactions between many genomic loci, and are well suited for identifying potential transcription clusters (Figure 6).

Using the initial criteria of chromatin accessibility and RNA Pol II binding, we identified 16,080 and 16,527 potential transcription clusters from fibroblasts and B lymphocytes, respectively (Table 1, Materials and Methods). The majority of these clusters involved at least one expressed gene (72.2\% in fibroblasts, $90.5 \%$ in B lymphocytes) and many involved at least two expressed genes (31.2\% in fibroblasts, $58.7 \%$ in B lymphocytes). While investigating the colocalization of expressed genes in transcription clusters, we found that over $30 \%$ of clusters containing multiple expressed genes had common transcription factors based on binding motifs (31.0\% in fibroblasts, $33.1 \%$ in B lymphocytes) and that over half of these common transcription factors were master regulators ( $56.6 \%$ in fibroblasts, $74.7 \%$ in B lymphocytes). Two example transcription clusters derived from 3-way, 4-way, and 5-way contacts from both fibroblasts and B lymphocytes are shown in Figure 7. These example 
clusters contain at least two genes and have at least one common transcription factor.

We tested the criteria for potential transcription clusters for statistical significance (Materials and Methods). That is, we tested whether the identified transcription clusters are more likely to include genes, and if these genes were more likely to share common transcription factors, than random multi-way contacts. We found that the identified transcription clusters were significantly more likely to include $\geq 1$ gene and $\geq 2$ genes than random multi-way contacts $(p<0.01)$. In addition, transcription clusters containing $\geq 2$ genes were significantly more likely to have common transcription factors and common master regulators $(p<0.01)$. After testing all orders of multi-way transcription clusters together, we also tested the 3way, 4-way, 5-way, and 6-way (or more) cases individually. We found that all cases were statistically significant $(p<0.01$ ) except for clusters with common transcription factors or master regulators in the 6-way (or more) case for both fibroblasts and B lymphocytes. We hypothesize that these cases were not statistically significant due to the large number of loci, naturally leading to an increased overlap with genes. This increases the likelihood that at least two genes will have common transcription factors or master regulators. Approximately half of the transcription clusters with at least two genes with common transcription factors also contained at least one enhancer $(\sim 51 \%$ and $\sim 44 \%$ in fibroblasts and B lymphocytes, respectively), offering further support that these multi-way contacts represented real transcription clusters [16].

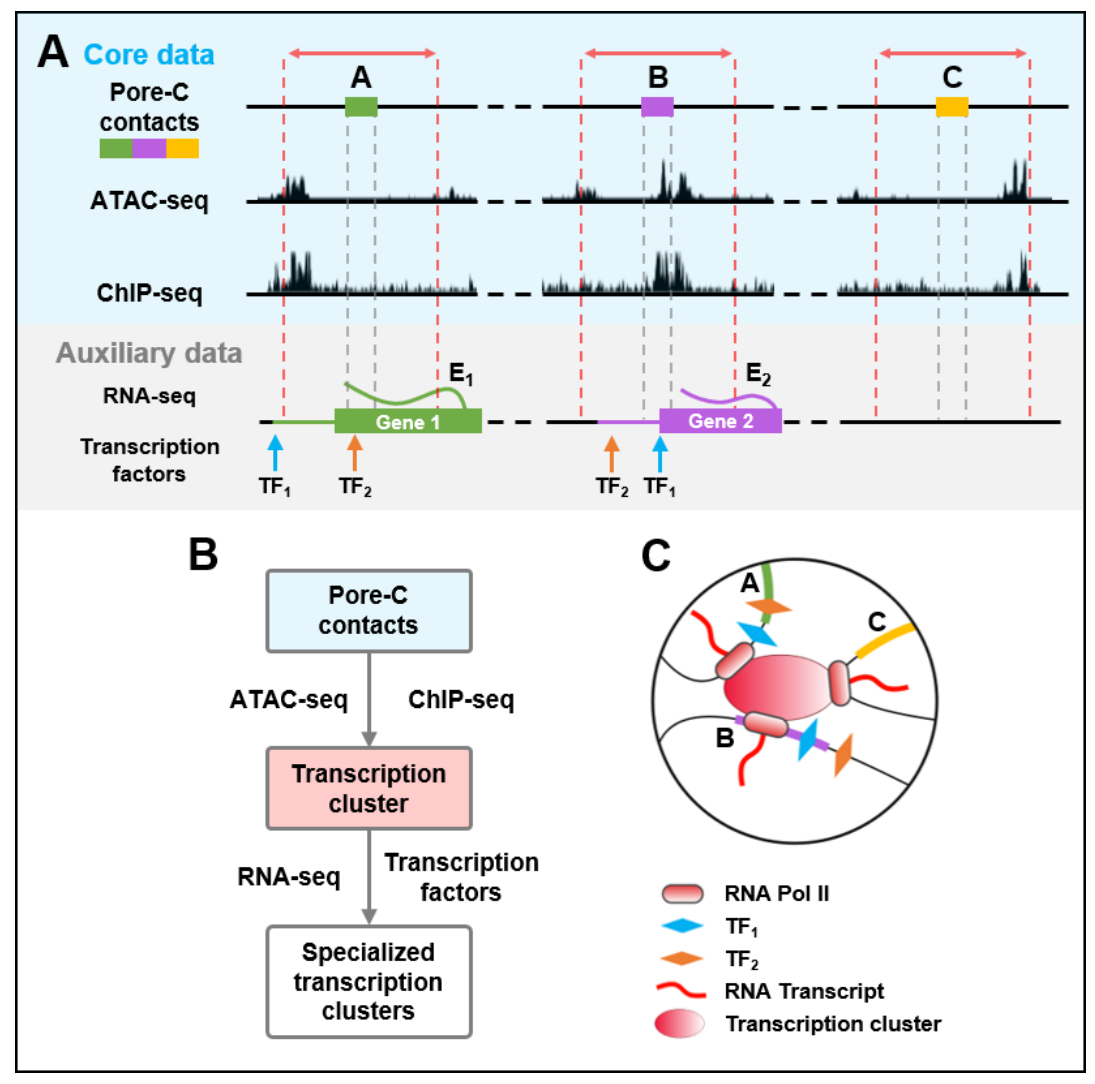

Figure 6: Data-driven identification of transcription clusters. (A) A $5 \mathrm{~kb}$ region before and after each locus in a Pore-C read (between red dashed lines) is queried for chromatin accessibility and RNA Pol II binding (ATAC-seq and ChIP-seq, respectively). Multi-way contacts between accessible loci that have $\geq 1$ instance of Pol II binding are indicative of potential transcription clusters. Gene expression (RNA-seq, E1 for gene 1 and E2 for gene 2, respectively) and transcription factor binding sites (TF1 and TF2) are integrated to determine potential coexpression and coregulation within multi-way contacts with multiple genes. Transcription factor binding sites are queried $\pm 5 \mathrm{~kb}$ from the gene's transcription start site (Materials and Methods). (B) Pipeline for extracting transcription clusters (Supplementary Materials). Genes are colored based on the overlapping Pore-C locus, and the extended line from each gene represents the 5 kb flanking region used to query transcription factor binding sites. (C) Schematic representation of a transcription cluster. 
bioRxiv preprint doi: https://doi.org/10.1101/2021.08.15.456363; this version posted September 2, 2021. The copyright holder for this preprint (which was not certified by peer review) is the author/funder. All rights reserved. No reuse allowed without permission.

\section{Fibroblast}

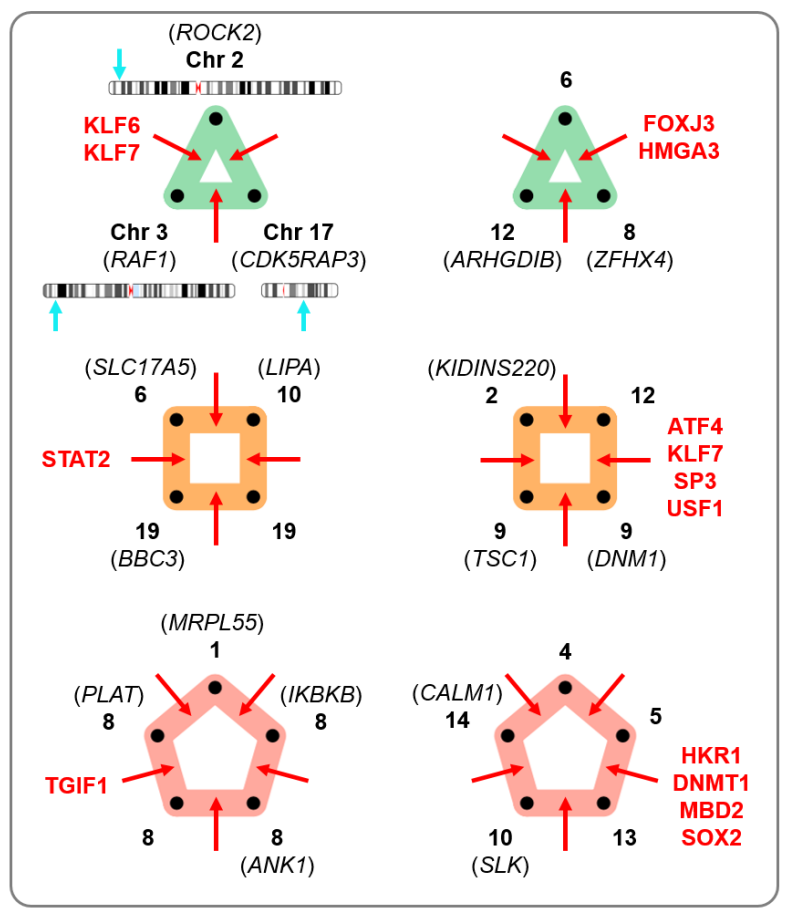

\section{B Lymphocyte}

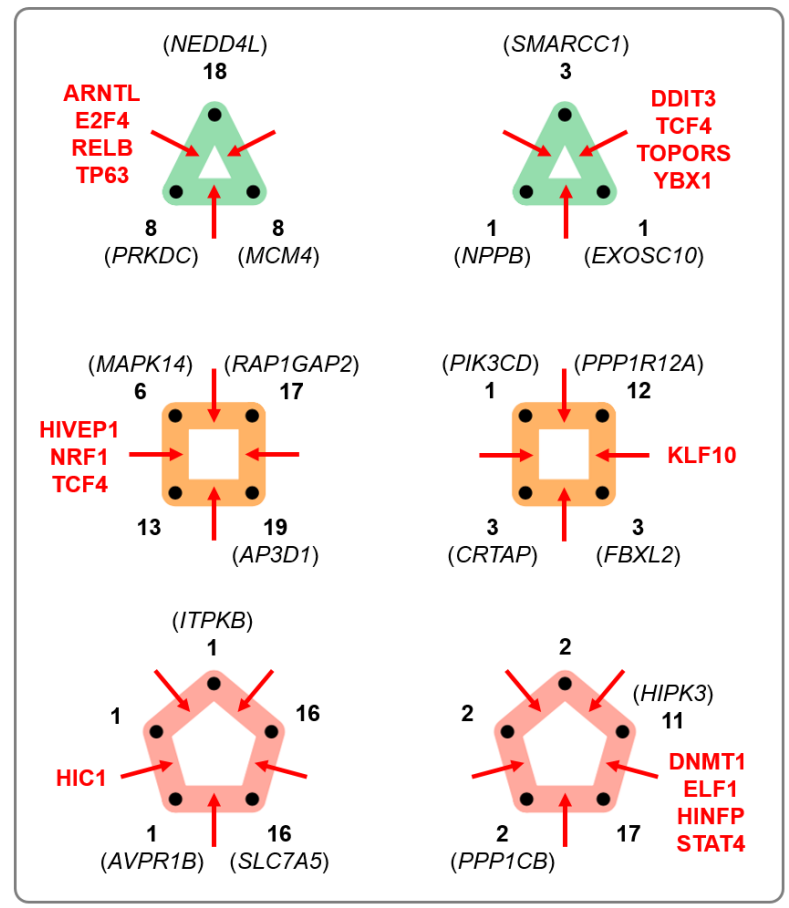

Figure 7: Example transcription clusters. Six examples of potential transcription clusters are shown for fibroblasts (left) and B lymphocytes (right) as multi-way contact motifs. Black labels indicate genes and chromosomes (bold). Red labels correspond to transcription factors shared between $\geq 2$ genes within a transcription cluster. Blue arrows indicate a gene's position on its respective chromosome. Multi-way contacts used for fibroblasts include all experiments (V1-V4). Examples were selected from the set of multi-way contacts summarized in the "Clusters with Common TFs" column of Table 1. 
bioRxiv preprint doi: https://doi.org/10.1101/2021.08.15.456363; this version posted September 2, 2021. The copyright holder for this preprint (which was not certified by peer review) is the author/funder. All rights reserved. No reuse allowed without permission.

\begin{tabular}{|c|c|c|c|c|c|c|}
\hline Order & $\begin{array}{c}\text { Multi-way } \\
\text { Contacts }\end{array}$ & $\begin{array}{c}\text { Transcription } \\
\text { Clusters }\end{array}$ & $\begin{array}{c}\text { Clusters with } \\
\mathbf{1} \text { 1 Gene }\end{array}$ & $\begin{array}{c}\text { Clusters with } \\
\geq \text { 2 Genes }\end{array}$ & $\begin{array}{c}\text { Clusters with } \\
\text { Common TFs }\end{array}$ & $\begin{array}{c}\text { Clusters with } \\
\text { Common MRs }\end{array}$ \\
\hline \multirow{2}{*}{3} & 379,165 & 11,261 & 7,782 & 2,986 & 1,191 & 679 \\
\cline { 2 - 7 } & 240,477 & 8,384 & 7,384 & 4,157 & 2,006 & 1,536 \\
\hline \multirow{2}{*}{4} & 181,554 & 3,254 & 2,519 & 1,214 & 276 & 153 \\
\cline { 2 - 7 } & 227,352 & 4,345 & 3,972 & 2,686 & 822 & 606 \\
\hline \multirow{2}{*}{5} & 98,272 & 1,021 & 831 & 473 & 63 & 35 \\
\cline { 2 - 7 } & 196,423 & 1,996 & 1,881 & 1,434 & 277 & 193 \\
\hline \multirow{2}{*}{$6+$} & 142,575 & 544 & 477 & 341 & 24 & 13 \\
\cline { 2 - 7 } & $1,000,231$ & 1,802 & 1,727 & 1,419 & 109 & 67 \\
\hline
\end{tabular}

Table 1: Summary of multi-way contacts. Multi-way contacts from fibroblasts (gray rows, V1-V4) and B lymphocytes (white rows) are listed after different filtering criteria. Multi-way contacts are considered to be potential transcription clusters if all loci within the multi-way contact are accessible and at least one locus has binding from RNA Pol II. These multi-way contacts are then queried for nearby expressed genes. If a transcription cluster candidate has at least two expressed genes, we determine whether the genes have common transcription factors (TFs) through binding motifs. From the set of transcription clusters with common transcription factors, we calculate how many clusters are regulated by at least one master regulator (MR).

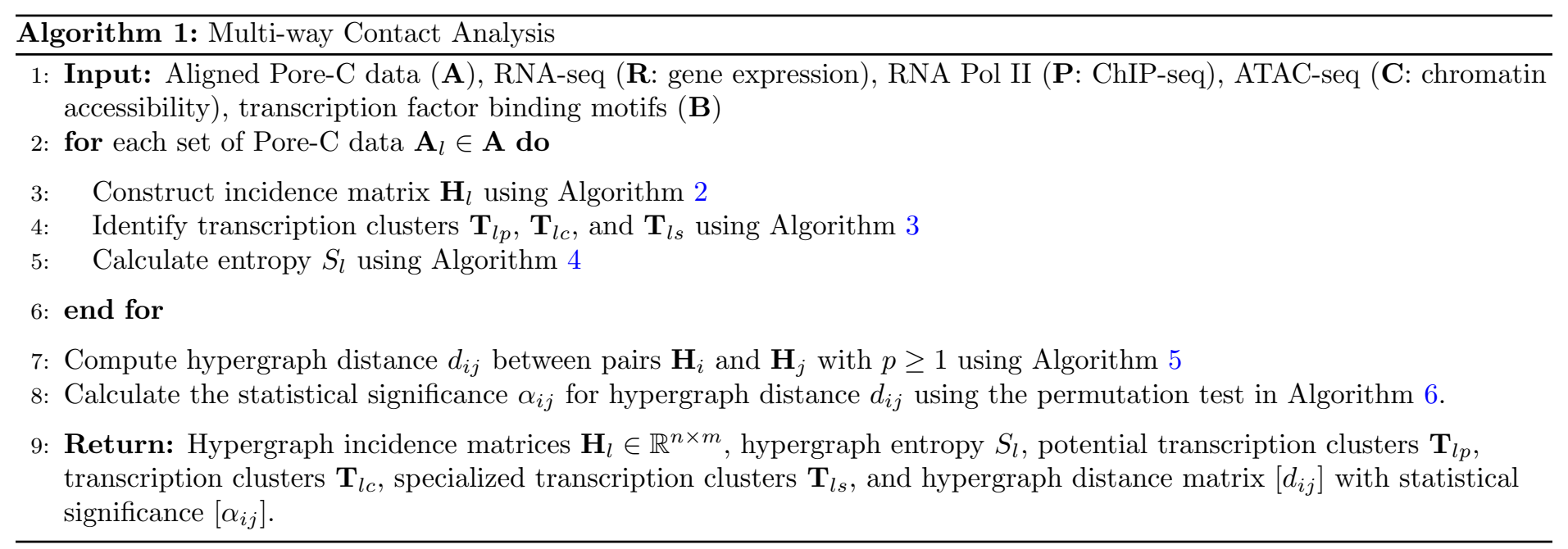

\section{Discussion}

In this work, we introduce a hypergraph framework to study higher-order chromatin structure from long-read sequence data. We demonstrate that multidimensional genomic architecture can be precisely represented and analyzed using hypergraph theory. Hypergraph representations strengthen and extend existing chromatin analysis techniques for study of TADs and intra- and inter-chromosomal interactions. The combination of long-read technology and accurate mathematical representations enable higher fidelity capture of the experimental system and deepen our understanding of genome architecture. Using direct capture of multi-way contacts, we identified transcriptional clusters with physical proximity and coordinated gene expression. Our framework thus enables study of explicit structure-function relationships that are observed directly from data, eliminating the need for inference of multi-way contacts. The increased precision of hypergraph representation has the potential to reveal patterns of higher-order differential chromatin organization between multiple cell-types, and presents the exciting possibility of application at the single cell-level $[6,7,8,17]$.

\section{Materials and Methods}

Cell Culture. Primary human adult dermal fibroblasts were obtained from a donor and were maintained in Dulbecco's Modified Eagle Medium (DMEM) supplemented with 10\% fetal bovine serum (FBS), 1X Glutamax (Thermo Fisher Scientific cat no. 35050061) and $1 \mathrm{X}$ nonessential amino acid (Thermo Fisher Scientific cat no. 11140050).

Cross-linking. Protocols for cross-linking were based on Ulahannan et al. [5]. 2.5 million cells were washed three times in chilled $1 \mathrm{X}$ phosphate buffered saline (PBS) in a $50 \mathrm{~mL}$ centrifuge tube, pelleted by centrifugation at $500 \mathrm{x} \mathrm{g}$ for $5 \mathrm{~min}$ at 
$4^{\circ} \mathrm{C}$ between each wash. Cells were resuspended in $10 \mathrm{~mL}$ room temperature 1X PBS $1 \%$ formaldehyde (Fisher Scientific cat no. BP531-500) by gently pipetting with a wide bore tip, then incubated at room temperature for 10 min. To quench the cross-linking reaction $527 \mu \mathrm{L}$ of $2.5 \mathrm{M}$ glycine was added to achieve a final concentration of $1 \% \mathrm{w} / \mathrm{v}$ or $125 \mathrm{mM}$ in $10.5 \mathrm{~mL}$. Cells were incubated for $5 \mathrm{~min}$ at room temperature followed by $10 \mathrm{~min}$ on ice. The cross-linked cells were pelleted by centrifugation at $500 \mathrm{xg}$ for $5 \mathrm{~min}$ at $4^{\circ} \mathrm{C}$.

Restriction Enzyme Digest. The cell pellet was resuspended in $500 \mu \mathrm{L}$ of cold permeabilization buffer $(10 \mathrm{mM}$ Tris-HCl $\mathrm{pH}$ 8.0, $10 \mathrm{mM} \mathrm{NaCl}, 0.2 \%$ IGEPAL CA-630, $100 \mu \mathrm{L}$ of protease inhibitor cock-tail Roche cat no. 11836170001) and placed on ice for $15 \mathrm{~min}$. One tablet of protease inhibitor cocktail was dissolved in $1 \mathrm{ml}$ nuclease free water and $100 \mu \mathrm{L}$ from that was added to a $500 \mu \mathrm{L}$ permeabilization buffer. Cells were centrifuged at $500 \mathrm{x} \mathrm{g}$ for $10 \mathrm{~min}$ at $4^{\circ} \mathrm{C}$ after which the supernatant was aspirated and replaced with $200 \mu \mathrm{L}$ of chilled 1.5X New England Biolabs (NEB) cutsmart buffer. Cells were centrifuged again at $500 \mathrm{x} \mathrm{g}$ for $10 \mathrm{~min}$ at $4^{\circ} \mathrm{C}$, then aspirated and re-suspended in $300 \mu \mathrm{L}$ of chilled 1.5X NEB cutsmart buffer. To denature the chromatin, $33.5 \mu \mathrm{L}$ of $1 \% \mathrm{w} / \mathrm{v}$ sodium dodecyl sulfate (SDS, Invitrogen cat no. 15553-035) was added to the cell suspension and incubated for exactly $10 \mathrm{~min}$ at $65^{\circ} \mathrm{C}$ with gentle agitation then placed on ice immediately afterwards. To quench the SDS, $37.5 \mu \mathrm{L}$ of 10\% v/v Triton X-100 (Sigma Aldrich cat no. T8787-250) was added for a final concentration of $1 \%$, followed by incubation for $10 \mathrm{~min}$ on ice. Permeabilized cells were then digested with a final concentration of $1 \mathrm{U} / \mathrm{\mu L}$ of NlaIII (NEB-R0125L) and brought to volume with nuclease-free water to achieve a final 1X digestion reaction buffer in $450 \mathrm{\mu L}$. Cells were then mixed by gentle inversion. Cell suspensions were incubated in a thermomixer at $37^{\circ} \mathrm{C}$ for 18 hours with periodic rotation.

Proximity Ligation and Reverse Cross-linking. NlaIII restriction digestion was heat inactivated at $65^{\circ} \mathrm{C}$ for $20 \mathrm{~min}$. Proximity ligation was set up at room temperature with the addition of the following reagents: $100 \mu \mathrm{L}$ of $10 \mathrm{X}$ T4 DNA ligase buffer (NEB), $10 \mu \mathrm{L}$ of $10 \mathrm{mg} / \mathrm{mL}$ BSA and $50 \mu \mathrm{L}$ of T4 Ligase (NEB M0202L) in a total volume of $1000 \mu \mathrm{L}$ with nuclease-free water. The ligation was cooled to $16^{\circ} \mathrm{C}$ and incubated for 6 hours with gentle rotation.

Protein Degradation and DNA Purification. To reverse cross-link, proximity ligated sample was treated with $100 \mu \mathrm{L}$ Proteinase K (NEB P8107S-800U/ml), $100 \mu \mathrm{L}$ 10\% SDS (Invitrogen cat no. 15553-035) and $500 \mu \mathrm{L} \mathrm{20 \%} \mathrm{v/v} \mathrm{Tween-20} \mathrm{(Sigma}$ Aldrich cat no. P1379) in a total volume of $2000 \mu \mathrm{L}$ with nuclease-free water. The mixture was incubated in a thermal block at $56^{\circ} \mathrm{C}$ for 18 hours. In order to purify DNA, the sample was transferred to a $15 \mathrm{~mL}$ centrifuge tube, rinsing the original tube with a further $200 \mu \mathrm{L}$ of nuclease-free water to collect any residual sample, bringing the total sample volume to $2.2 \mathrm{~mL}$. DNA was then purified from the sample using a standard phenol chloroform extraction and ethanol precipitation.

Nanopore Sequencing. Purified DNA was Solid Phase Reversible Immobilization (SPRI) size selected before library preparation with a bead ratio of $0.48 \mathrm{X}$ for fragments $>1.5 \mathrm{~kb}$. The $>1.5 \mathrm{~kb}$ products were prepared for sequencing using the protocol provided by Oxford Nanopore Technologies. In brief, $1 \mathrm{\mu g}$ of genomic DNA input was used to generate a sequencing library according to the protocol provided for the SQK-LSK109 kit (Oxford Nanopore Technologies, Oxford Science Park, UK, version GDE_9063_v109_revU_14Aug2019). After the DNA repair, end prep, and adapter ligation steps, SPRI select bead suspension (Cat No. B23318, Beckman Coulter Life Sciences, Indianapolis, IN, USA) was used to remove short fragments and free adapters. A bead ratio of $1 \mathrm{X}$ was used for DNA repair and end prep while a bead ratio of $0.4 \mathrm{X}$ was used for the adapter ligation step. Qubit dsDNA assay (ThermoFisher Scientific, Waltham, MA, USA) was used to quantify DNA and $\sim 300-400 \mathrm{ng}$ of DNA library was loaded onto a GridION flow cell (version R9, Flo-MIN 106D). In total, 4 sequencing runs were conducted generating a total of 6.25 million reads (referred to as V1-V4).

Sequence Processing. Reads which passed Q-score filtering (--min_qscore 7, 4.56 million reads) after base calling on the Oxford Nanopore GridION (Guppy, version 4.0.11) were used as input for the Pore-C-Snakemake pipeline (https://github . $\mathrm{com} /$ nanoporetech/Pore-C-Snakemake, commit 6b2f762). The pipeline maps multi-way contacts to a reference genome and stores the hyperedge data in a variety of formats. The reference genome used for mapping was GRCh38.p13 (https: //www.ncbi.nlm.nih.gov/assembly/GCF_000001405.39/). Each of the four sequencing runs were assigned a sequencing run label and then concatenated. The combined pipeline outputs were used as standard inputs for all downstream analysis.

Hypergraphs. A hypergraph is a generalization of a graph. Hypergraphs are composed of hyperedges, which can join any number of nodes [18]. Hypergraphs can capture higher order connectivity patterns and represent multidimensional relationships unambiguously $[6,19]$. In hypergraphs obtained from Pore-C data, we defined nodes as genomic loci at a particular resolution (e.g. read level, $100 \mathrm{~kb}, 1 \mathrm{Mb}$, or $25 \mathrm{Mb}$ bins), and hyperedges as contacts among genomic loci. Most higher order contacts are unique in Pore-C data at high resolution (read level or $100 \mathrm{~kb}$ ), so for these data we considered unweighted hypergraphs (i.e. ignore the frequency of contacts). For lower resolutions (1 Mb or $25 \mathrm{Mb}$ ), we considered edge weights (frequency of contacts) to find the most common intra- and inter-chromosomal contacts.

Hypergraph Filtering. We performed an additional filtering step while constructing genomic hypergraphs. We first decomposed each multi-way contact into its pairwise combinations at a particular resolution. From these pairwise contacts, 
we counted the number of times a contact was detected for a each pair of loci, and identified the highest frequency locus pairs. Pairwise contacts were kept if they occurred above a certain threshold number, which was set empirically at the $85^{\text {th }}$ percentile of the most frequently occurring locus pairs. For example, in fibroblast data binned at $1 \mathrm{Mb}$ resolution, a locus pair with six detected contacts corresponded to the $85^{\text {th }}$ percentile. Thus all pairs of loci with fewer than six detected contacts were not considered, which increases confidence in the validity of identified multi-way contacts.

Incidence Matrices. An incidence matrix of the genomic hypergraph was an $n$-by- $m$ matrix containing values zero and one. The row size $n$ was the total number of genomic loci, and the column size $m$ was the total number of unique Pore-C contacts (including self-contacts, pairwise contacts, and higher order contacts). Nonzero elements in a column of the incidence matrix indicate genomic loci contained in the corresponding Pore-C contact. Thus, the number of nonzero elements gave the order of the Pore-C contact. The incidence matrix of the genomic hypergraph can be visualized via PAOHvis [20]. In PAOHvis, genomic loci are parallel horizontal bars, while Pore-C contacts are vertical lines that connects multiple loci (see Figures 1, 2, 3, and 4). Beyond visualization, incidence matrices play a significant role in the mathematical analysis of hypergraphs.

Data-driven Identification of Transcription Clusters. We used Pore-C data in conjunction with multiple other data sources to identify potential transcription clusters (Figure 6). Each locus in a Pore-C read, or multi-way contact, was queried for chromatin accessibility and RNA Pol II binding (ATAC-seq and ChIP-seq peaks, respectively). Multi-way contacts were considered to be potential transcription clusters if all loci within the multi-way contact are accessible and at least one locus had binding of RNA Pol II. The loci in potential transcription clusters were then queried for nearby expressed genes. A $5 \mathrm{~kb}$ flanking region was added before and after each locus when querying for chromatin accessibility, RNA Pol II binding, and nearby genes [21]. Gene expression (RNA-seq) and transcription factor binding sites were then integrated to determine coexpression and coregulation of genes in transcription clusters. If a transcription cluster candidate had at least two genes present, we determined whether the genes had common transcription factors through binding motifs. From the set of transcription clusters with common transcription factors, we calculated how many clusters were regulated by at least one master regulator, a transcription factor that also regulates its own gene (Figure 6).

Transcription Factor Binding Motifs. Transcription factor binding site motifs were obtained from "The Human Transcription Factors" database [22]. FIMO (https://meme-suite.org/meme/tools/fimo) was used to scan for motifs within \pm $5 \mathrm{~kb}$ of genes' transcription start sites. The results were converted to a 22,083 $\times 1,007$ MATLAB table, where rows were genes, columns were transcription factors, and entries were the number of binding sites for a particular transcription factor and gene. The table was then filtered to only include entries with three or more binding sites in downstream computations. This threshold was determined empirically and is adjustable in the MATLAB code.

Public Data Sources. Pore-C data for B lymphocytes were downloaded from Ulahannan et al. [5]. ATAC-seq and ChIP-seq data were obtained from the Encyclopedia of DNA Elements (ENCODE) to assess chromatin accessibility and RNA Pol II binding, respectively. These data were compared to read-level Pore-C contacts to determine whether colocalizing loci belong to accessible regions of chromatin and had RNA Pol II binding for both fibroblasts and B lymphocytes. RNA-seq data were also obtained from ENCODE to ensure that genes within potential transcription factories were expressed in their respective cell types. A summary of these data sources can be found in Table 2.

\begin{tabular}{|c|c|l|}
\hline Data Type & Cell Type & \multicolumn{1}{|c|}{ Data Description and Source } \\
\hline Pore-C & Fibroblasts & Human primary dermal fibroblasts were derived from a donor skin biopsy \\
\hline Pore-C & GM12878 & B-lymphocyte Pore-C data obtained from Ulahannan et al. $[5]$ \\
\hline ATAC-seq & IMR-90 & Fibroblast chromatin accessibility (ENCFF310UDS) \\
\hline ATAC-seq & GM12878 & B-lymphocyte chromatin accessibility data (ENCFF410XEP) \\
\hline ChIP-seq & IMR-90 & Fibroblast RNA Polymerase II binding data (ENCFF676DGR) \\
\hline ChIP-seq & GM12878 & B-lymphocyte RNA Polymerase II binding data (ENCFF912DZY) \\
\hline RNA-seq & IMR-90 & Fibroblast gene expression data averaged over two replicates (ENCFF353SBP, ENCFF496RIW) \\
\hline RNA-seq & GM12878 & B-lymphocyte gene expression data averaged over two replicates (ENCFF306TLL, ENCFF418FIT) \\
\hline Enhancers & IMR-90 & Fibroblast enhancer location data from EnhancerAtlas 2.0 [16] \\
\hline Enhancers & GM12878 & B-lymphocyte enhancer location data from EnhancerAtlas 2.0 [16] \\
\hline
\end{tabular}

Table 2: Data sources. Data obtained from ENCODE unless otherwise specified [23].

Hypergraph Entropy. Network entropy often is used to measure the connectivity and regularity of a network [8, 24, 25]. We used the hypergraph entropy proposed in [26] to quantify the organization of chromatin structure from Pore-C data. 
Denote the incidence matrix of the genomic hypergraph by $\mathbf{H}$. The Laplacian matrix then is an $n$-by- $n$ matrix $(n$ is the total number of genomic loci in the hypergraph), which can be computed by $\mathbf{L}=\mathbf{H H}^{\top} \in \mathbb{R}^{n \times n}$, where $\top$ denotes matrix transpose. Therefore, the hypergraph entropy is defined by

$$
\text { Hypergraph Entropy }=-\sum_{i=1}^{n} \lambda_{i} \ln \lambda_{i}
$$

where $\lambda_{i}$ are the normalized eigenvalues of $\mathbf{L}$ such that $\sum_{i=1}^{n} \lambda_{i}=1$, and the convention $0 \ln 0=0$ is used. In mathematics, eigenvalues can quantitatively represent different features of a matrix [27]. Biologically, genomic regions with high entropy are likely associated with high proportions of euchromatin (i.e. less organized folding patterns), as euchromatin is more structurally permissive than heterochromatin [28, 29, 30].

We computed the entropy of intra-chromosomal genomic hypergraphs for both fibroblasts and B lymphocytes as shown in Figure S2. It is expected that larger chromosomes have larger hypergraph entropy because more potential genomic interactions occur in the large chromosomes. However, there are still subtle differences between the fibroblast and B lymphocyte chromosomes, indicating differences in their genome structure. In order to better quantify the structure properties of chromosomes and compare between cell types, it may be useful to introduce normalizations to hypergraph entropy in the future.

Hypergraph Distance. Comparing graphs is a ubiquitous task in data analysis and machine learning [31, 31, 32, 33]. We propose a spectral-based hypergraph distance measure which can be used to quantify global difference between two genomic hypergraphs $\mathrm{G}_{1}$ and $\mathrm{G}_{2}$. Denote the incidence matrices of two genomic hypergraphs by $\mathbf{H}_{1} \in \mathbb{R}^{n \times m_{1}}$ and $\mathbf{H}_{2} \in \mathbb{R}^{n \times m_{2}}$, respectively. For $i=1,2$, construct the normalized Lapalacian matrices:

$$
\tilde{\mathbf{L}}_{i}=\mathbf{I}-\mathbf{D}_{i}^{-\frac{1}{2}} \mathbf{H}_{i} \mathbf{E}_{i}^{-1} \mathbf{H}_{i}^{\top} \mathbf{D}_{i}^{-\frac{1}{2}} \in \mathbb{R}^{n \times n}
$$

where $\mathbf{I} \in \mathbb{R}^{n \times n}$ is the identity matrix, $\mathbf{E}_{i} \in \mathbb{R}^{m_{i} \times m_{i}}$ is a diagonal matrix containing the orders of hyperedges along its diagonal, and $\mathbf{D}_{i} \in \mathbb{R}^{n \times n}$ is a diagonal matrix containing the degrees of nodes along its diagonal [34]. The degree of a node is equal to the number of hyperedges that contain that node. Therefore, the hypergraph distance between $G_{1}$ and $G_{2}$ is defined by

$$
\text { Hypergraph } \operatorname{Distance}\left(\mathrm{G}_{1}, \mathrm{G}_{2}\right)=\frac{1}{n}\left(\sum_{i=1}^{n}\left|\lambda_{1 j}-\lambda_{2 j}\right|^{p}\right)^{\frac{1}{p}} \text {, }
$$

where $\lambda_{i j}$ is the $j$ th eigenvalue of $\tilde{\mathbf{L}}_{i}$ for $i=1,2$, and $p \geq 1$. In our analysis, we choose $p=2$. The hypergraph distance $(3)$ can be used to compare two genomic hypergraphs in a global scale since the eigenvalues of the normalized Laplacian are able to capture global connectivity patterns within the hypergraph.

We computed the distance between two genome-wide hypergraphs derived from fibroblasts and B lymphocytes, and examined the distance statistically through a permutation test. Figure S3A demonstrates that the two genomic hypergraphs are significantly different, with a $p$-value $<0.01$. Additionally, we computed the distance between intra-chromosomal genomic hypergraphs between fibroblasts and B lymphocytes. We found that Chromosome 19 and 21 have the largest distances between cell types, as seen in Figure S3B.

Statistical Significance via Permutation Test. In order to assess the statistical significance of the transcription cluster candidates we determined using our criteria (Figure 6), we used a permutation test which builds the shape of the null hypothesis (i.e. the random background distribution) by resampling the observed data over $N$ trials. We randomly selected $n$ 3rd, 4th, 5th, and 6th or more order multi-way contacts from our Pore-C data, where $n$ was based on the number of transcription cluster candidates we determined for each order. For example, we randomly selected $n=11,261$ multi-way contacts from the set of 3rd order multi-way contacts in fibroblasts (Table 1). For each trial, we determined how many of these randomly sampled "transcription clusters" match our remaining criteria: transcription clusters with $\geq 1$ gene, $\geq 2$ genes, common TFs, and common MRs. The background distribution for each of the criteria was then constructed from these values. The proportion of values in the background distributions that was greater than their counterparts from the dataderived transcription cluster candidates yielded the $p$-value. This analysis was based on the assumption that transcription clusters will be more likely to contain genes and that those genes are more likely to have common transcription factors than random multi-way contacts. For this analysis, we chose $N=1,000$ trials.

Similarly, we used a permutation test to determine the significance of the measured distances between two hypergraphs. Suppose that we are comparing two hypergraphs $\mathrm{G}_{1}$ and $\mathrm{G}_{2}$. We first randomly generate $N$ hypergraphs $\left\{\mathrm{R}_{i}\right\}_{i=1}^{N}$ that are similar to $G_{1}$ ("similar" means similar number of node degree and hyperedge size distribution). The background distribution therefore can be constructed by measuring the hypergraph distances between $\mathrm{G}_{1}$ and $\mathrm{R}_{i}$ for $i=1,2, \ldots, N$. The proportion of distances that was greater than the distance between $G_{1}$ and $G_{2}$ in this background distribution yielded the p-value. For this analysis, we again chose $N=1,000$ trials. 


\section{Code and Data Availability}

All data generated within this manuscript and MATLAB code in our computational framework can be provided upon request.

\section{Acknowledgements}

We thank Professor Peter Cook from the University of Oxford for his guidance and helpful discussions on transcription clusters. This work is supported in part by the Air Force Office of Scientific Research (AFOSR) award FA9550-18-1-0028 to IR and the Defense Advanced Research Projects Agency (DARPA) award 140D6319C0020 and the National Science Foundation (NSF) award 2035827 to iReprogram, LLC.

\section{Author Contributions}

IR designed research; SL, CC, SD, SJ, WM, AC, NB, CR, LM and IR performed research; SL, CC, SD, and IR contributed new reagents/analytic tools; SL, CC, SD, CR, CS, AC, AS and IR analyzed data; and SL, CC, SD, CS, NB, AS, MW, LM, IR wrote the paper.

\section{Competing Interests}

SD is an employee of iReprogram, LLC. LM and IR are co-founders of iReprogram, LLC. NB is an employee of Oxford Nanopore Technologies. SL, CC, SD, and IR have submitted a patent application for the computational framework (2115008250-US-PS1).

\section{References}

[1] Erez Lieberman-Aiden, Nynke L Van Berkum, Louise Williams, Maxim Imakaev, Tobias Ragoczy, Agnes Telling, Ido Amit, Bryan R Lajoie, Peter J Sabo, Michael O Dorschner, et al. Comprehensive mapping of long-range interactions reveals folding principles of the human genome. Science, 326(5950):289-293, 2009.

[2] Tom Misteli. The self-organizing genome: Principles of genome architecture and function. Cell, 183:28-45, 2020.

[3] Haiming Chen, Jie Chen, Lindsey A Muir, Scott Ronquist, Walter Meixner, Mats Ljungman, Thomas Ried, Stephen Smale, and Indika Rajapakse. Functional organization of the human 4d nucleome. Proceedings of the National Academy of Sciences, 112(26):8002-8007, 2015.

[4] Pedro Olivares-Chauvet, Zohar Mukamel, Aviezer Lifshitz, Omer Schwartzman, Noa Oded Elkayam, Yaniv Lubling, Gintaras Deikus, Robert P Sebra, and Amos Tanay. Capturing pairwise and multi-way chromosomal conformations using chromosomal walks. Nature, 540(7632):296-300, 2016.

[5] Netha Ulahannan, Matthew Pendleton, Aditya Deshpande, Stefan Schwenk, Julie M Behr, Xiaoguang Dai, Carly Tyer, Priyesh Rughani, Sarah Kudman, Emily Adney, et al. Nanopore sequencing of dna concatemers reveals higher-order features of chromatin structure. bioRxiv, page 833590, 2019.

[6] Austin R Benson, David F Gleich, and Jure Leskovec. Higher-order organization of complex networks. Science, 353(6295):163-166, 2016.

[7] Can Chen, Amit Surana, Anthony M. Bloch, and Indika Rajapakse. Controllability of hypergraphs. IEEE Transactions on Network Science and Engineering, 8(2):1646-1657, 2020.

[8] Can Chen and Indika Rajapakse. Tensor entropy for uniform hypergraphs. IEEE Transactions on Network Science and Engineering, 7(4):2889-2900, 2020.

[9] Jesse R Dixon, Siddarth Selvaraj, Feng Yue, Audrey Kim, Yan Li, Yin Shen, Ming Hu, Jun S Liu, and Bing Ren. Topological domains in mammalian genomes identified by analysis of chromatin interactions. Nature, 485(7398):376$380,2012$.

[10] Suhas SP Rao, Miriam H Huntley, Neva C Durand, Elena K Stamenova, Ivan D Bochkov, James T Robinson, Adrian L Sanborn, Ido Machol, Arina D Omer, Eric S Lander, et al. A 3d map of the human genome at kilobase resolution reveals principles of chromatin looping. Cell, 159(7):1665-1680, 2014.

[11] Jie Chen, Alfred O Hero III, and Indika Rajapakse. Spectral identification of topological domains. Bioinformatics, 32(14):2151-2158, 2016. 
[12] David M Suter, Nacho Molina, David Gatfield, Kim Schneider, Ueli Schibler, and Felix Naef. Mammalian genes are transcribed with widely different bursting kinetics. Science, 332(6028):472-474, 2011.

[13] Peter R Cook. The organization of replication and transcription. Science, 284(5421):1790-1795, 1999.

[14] Peter R Cook and Davide Marenduzzo. Transcription-driven genome organization: a model for chromosome structure and the regulation of gene expression tested through simulations. Nucleic Acids Research, 46(19):9895-9906, 2018.

[15] Cameron S Osborne, Lyubomira Chakalova, Karen E Brown, David Carter, Alice Horton, Emmanuel Debrand, Beatriz Goyenechea, Jennifer A Mitchell, Susana Lopes, Wolf Reik, et al. Active genes dynamically colocalize to shared sites of ongoing transcription. Nature Genetics, 36(10):1065-1071, 2004.

[16] Tianshun Gao and Jiang Qian. Enhanceratlas 2.0: an updated resource with enhancer annotation in 586 tissue/cell types across nine species. Nucleic Acids Research, 48(D1):D58-D64, 2020.

[17] Amit Surana, Can Chen, and Indika Rajapakse. Hypergraph dissimilarity measures. arXiv preprint arXiv:2106.08206, 2021.

[18] Claude Berge. Hypergraphs: combinatorics of finite sets, volume 45. Elsevier, 1984.

[19] Michael M Wolf, Alicia M Klinvex, and Daniel M Dunlavy. Advantages to modeling relational data using hypergraphs versus graphs. In 2016 IEEE High Performance Extreme Computing Conference (HPEC), pages 1-7. IEEE, 2016.

[20] Paola Valdivia, Paolo Buono, Catherine Plaisant, Nicole Dufournaud, and Jean-Daniel Fekete. Analyzing dynamic hypergraphs with parallel aggregated ordered hypergraph visualization. IEEE transactions on visualization and computer graphics, 27(1):1-13, 2019.

[21] Shane Neph, Andrew B Stergachis, Alex Reynolds, Richard Sandstrom, Elhanan Borenstein, and John A Stamatoyannopoulos. Circuitry and dynamics of human transcription factor regulatory networks. Cell, 150(6):1274-1286, 2012.

[22] Samuel A Lambert, Arttu Jolma, Laura F Campitelli, Pratyush K Das, Yimeng Yin, Mihai Albu, Xiaoting Chen, Jussi Taipale, Timothy R Hughes, and Matthew T Weirauch. The human transcription factors. Cell, 172(4):650-665, 2018.

[23] ENCODE Project Consortium et al. An integrated encyclopedia of dna elements in the human genome. Nature, 489(7414):57, 2012.

[24] Filippo Passerini and Simone Severini. The von neumann entropy of networks. arXiv:0812.2597, 2008.

[25] Giorgia Minello, Luca Rossi, and Andrea Torsello. On the von neumann entropy of graphs. Journal of Complex Networks, $7(4): 491-514,2019$.

[26] Isabelle Bloch and Alain Bretto. A new entropy for hypergraphs. In International Conference on Discrete Geometry for Computer Imagery, pages 143-154. Springer, 2019.

[27] Gilbert Strang. Introduction to linear algebra, volume 3. Wellesley-Cambridge Press Wellesley, MA, 1993.

[28] Ben D MacArthur and Ihor R Lemischka. Statistical mechanics of pluripotency. Cell, 154(3):484-489, 2013.

[29] Indika Rajapakse, Mark Groudine, and Mehran Mesbahi. What can systems theory of networks offer to biology? PLoS Computational Biology, 8(6):e1002543, 2012.

[30] Stephen Lindsly, Can Chen, Sijia Liu, Scott Ronquist, Samuel Dilworth, Michael Perlman, and Indika Rajapakse. 4dnvestigator: time series genomic data analysis toolbox. Nucleus, 12(1):58-64, 2021.

[31] Claire Donnat and Susan Holmes. Tracking network dynamics: A survey using graph distances. The Annals of Applied Statistics, 12(2):971 - 1012, 2018.

[32] Katherine Faust and John Skvoretz. Comparing networks across space and time, size and species. Sociological Methodology, 32(1):267-299, 2002.

[33] Peter Wills and François G Meyer. Metrics for graph comparison: a practitioner's guide. PloS One, 15(2):e0228728, 2020.

[34] Dengyong Zhou, Jiayuan Huang, and Bernhard Schölkopf. Learning with hypergraphs: Clustering, classification, and embedding. Advances in Neural Information Processing Systems, 19:1601-1608, 2006. 


\section{Supplementary Materials}
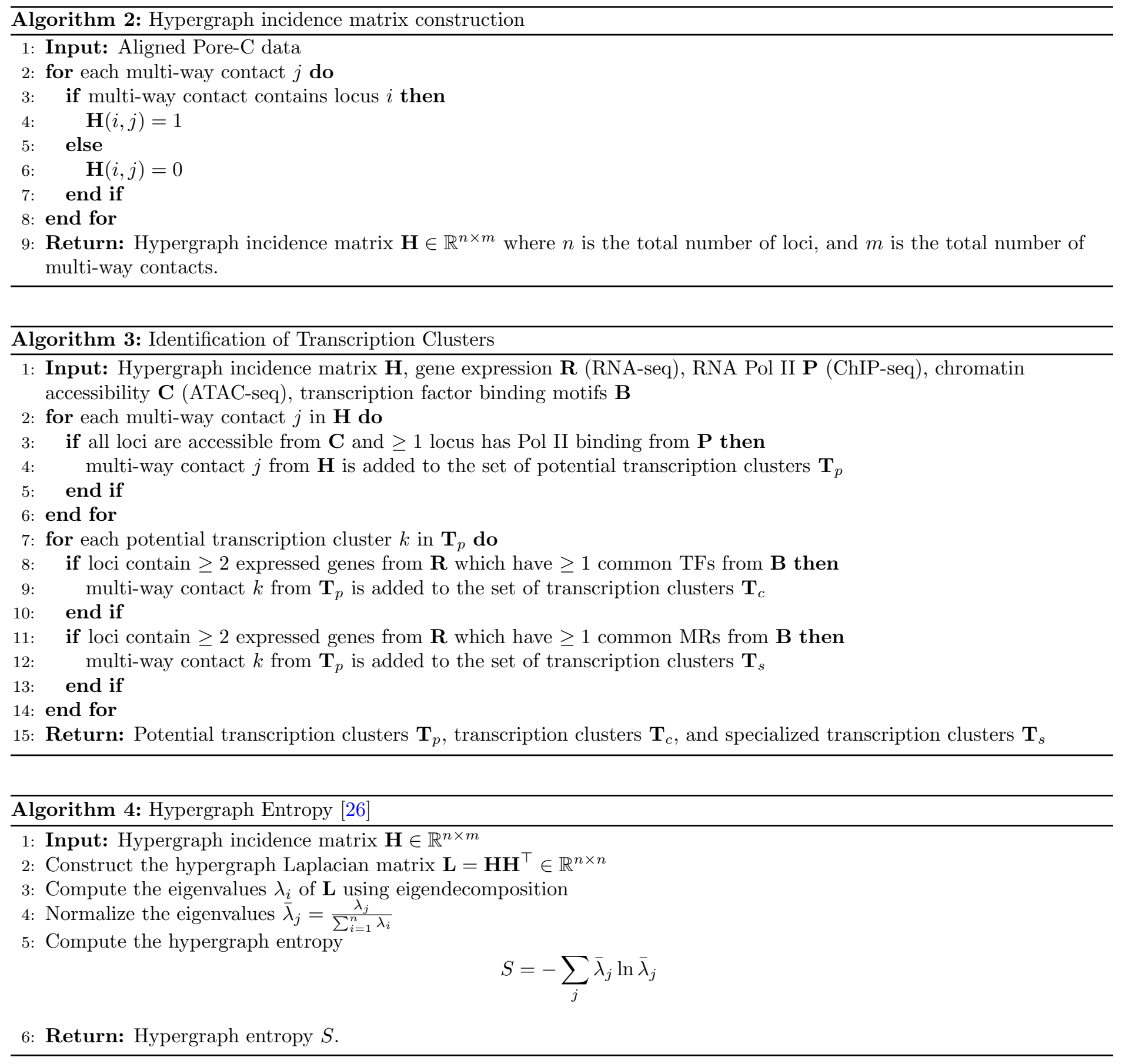

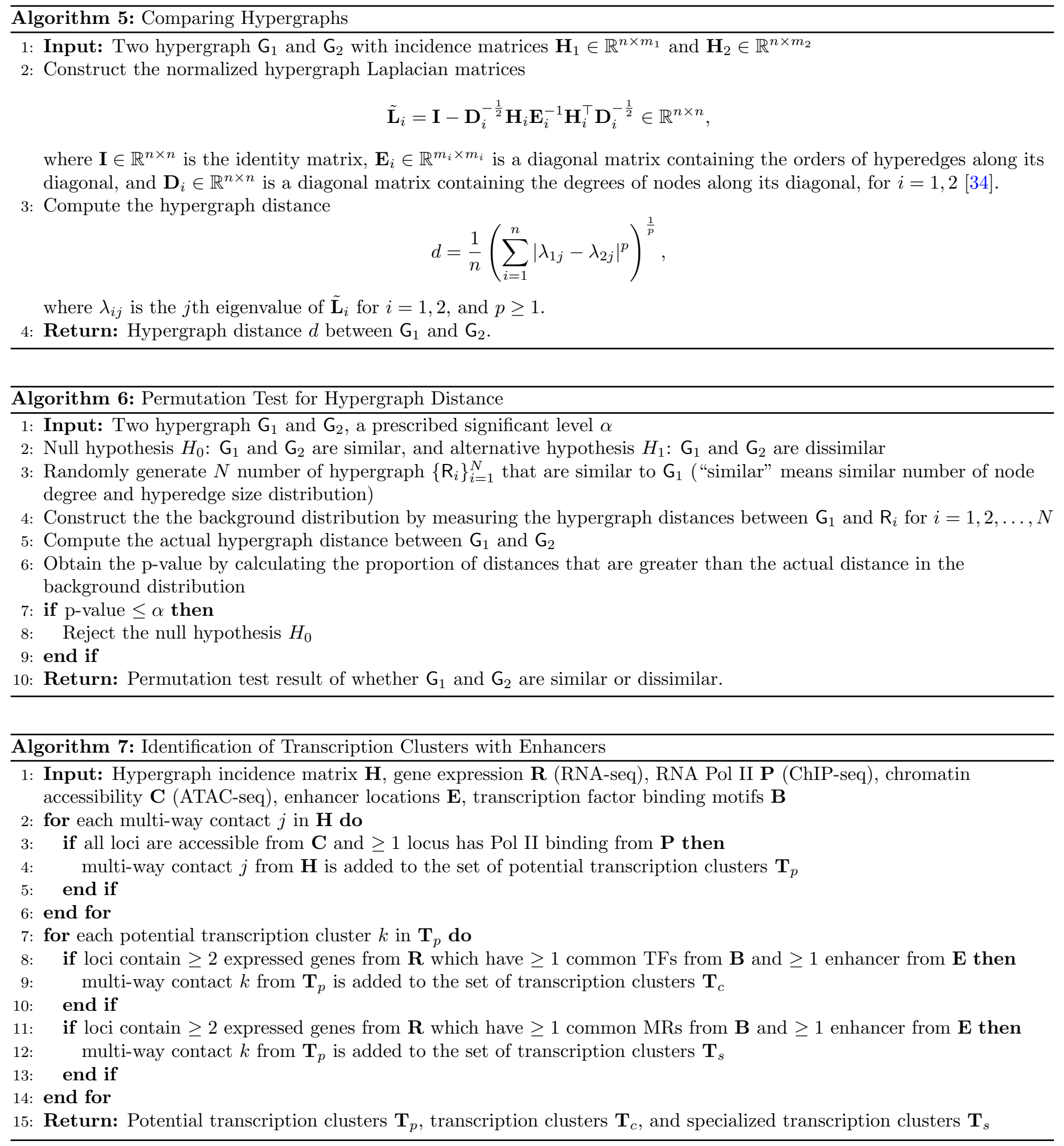
bioRxiv preprint doi: https://doi.org/10.1101/2021.08.15.456363; this version posted September 2, 2021. The copyright holder for this preprint (which was not certified by peer review) is the author/funder. All rights reserved. No reuse allowed without permission.

\section{Supplementary Figures}

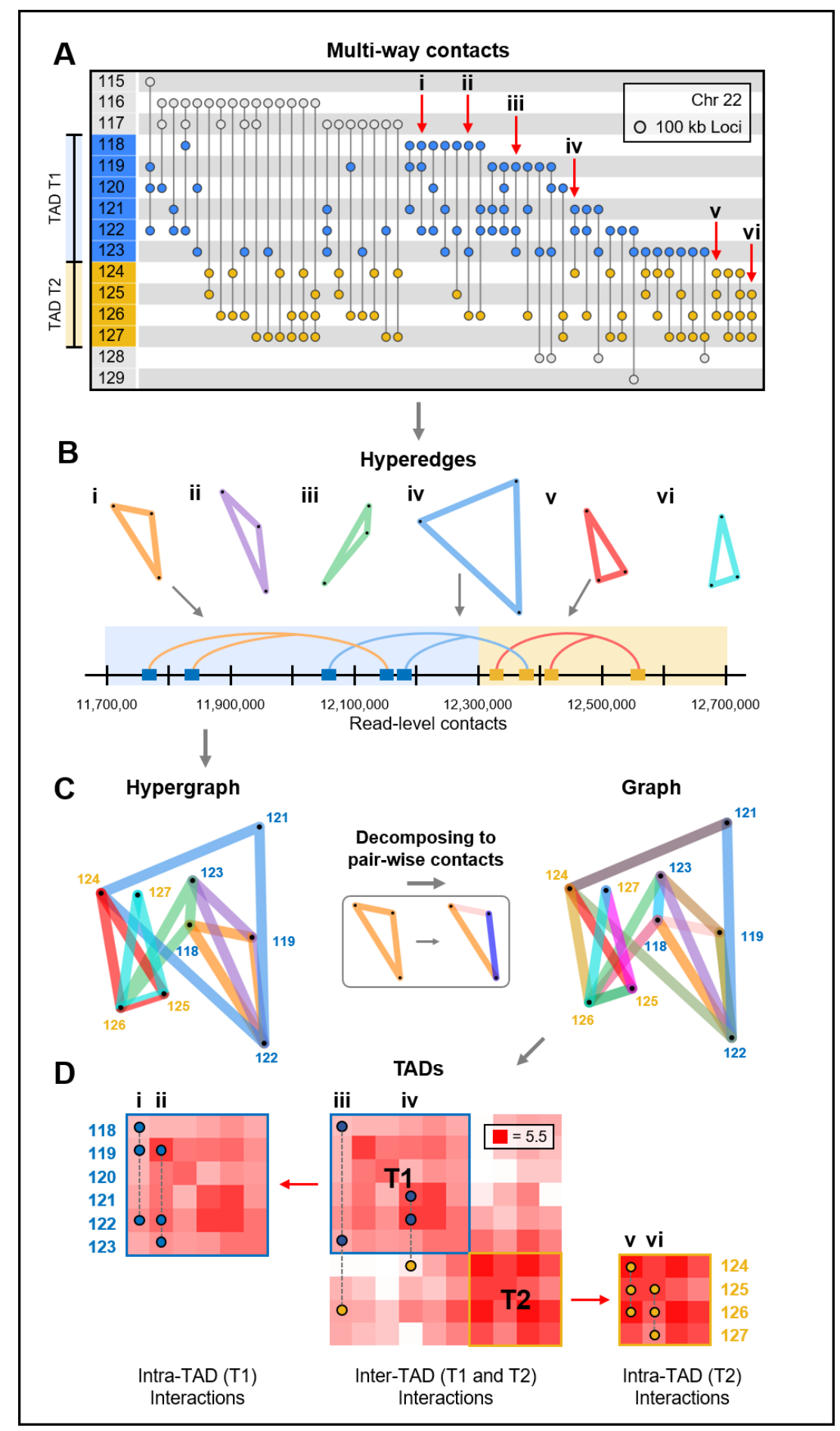

Figure S1: Local organization of the genome. (A) Incidence matrix visualization of a region in Chromosome 22 from B lymphocytes. The numbers in the left column represent genomic loci, vertical lines represent multi-way contacts, where nodes indicate the corresponding locus' participation in this contact. The blue and yellow regions represent two TADs, T1 and T2. The six contacts, denoted by the labels i-vi, are used as examples for hypergraph and genomic folding pattern visualizations. (B) Hypergraph visualization of the multi-way contacts i-vi from A. Blue and yellow labels indicate which TADs these loci participate in. (C) Contact frequency matrices were constructed by separating all multi-way contacts within and between the two TADs into their pairwise combinations. Example multi-way contacts are superimposed onto contact frequency matrices. All multi-way contacts in this figure were determined in $100 \mathrm{~kb}$ resolution after noise reduction (Materials and Methods). 


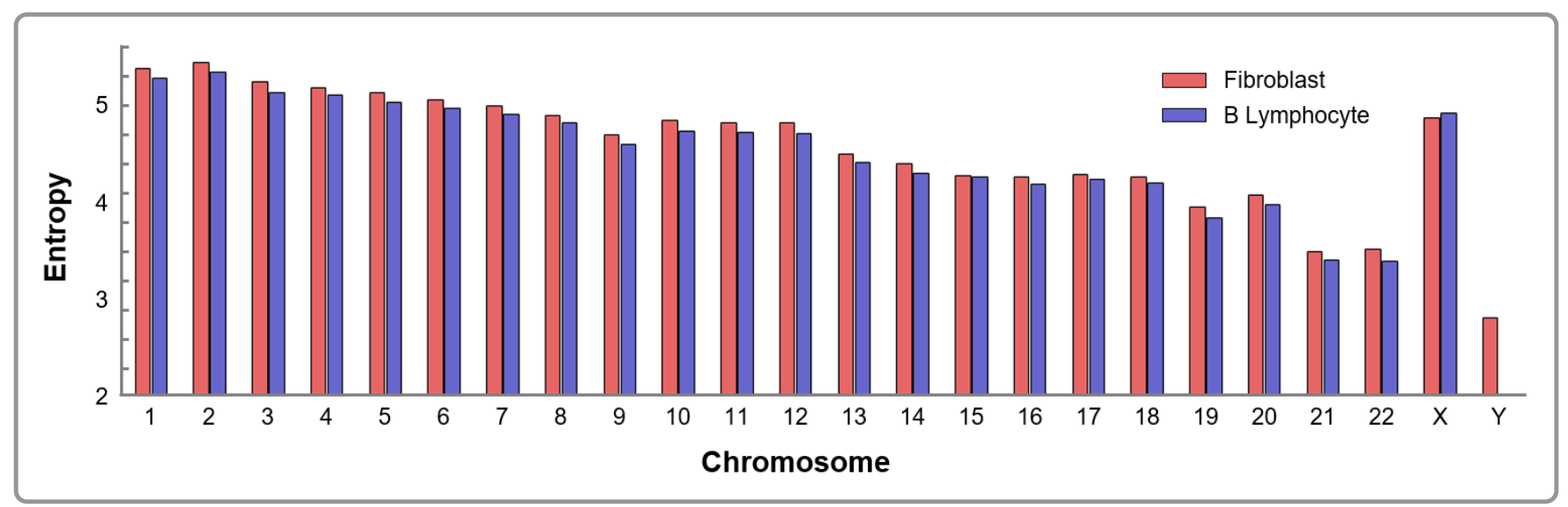

Figure S2: Entropy of intra-chromosomal genomic hypergraph for fibroblast and B lymphocytes.

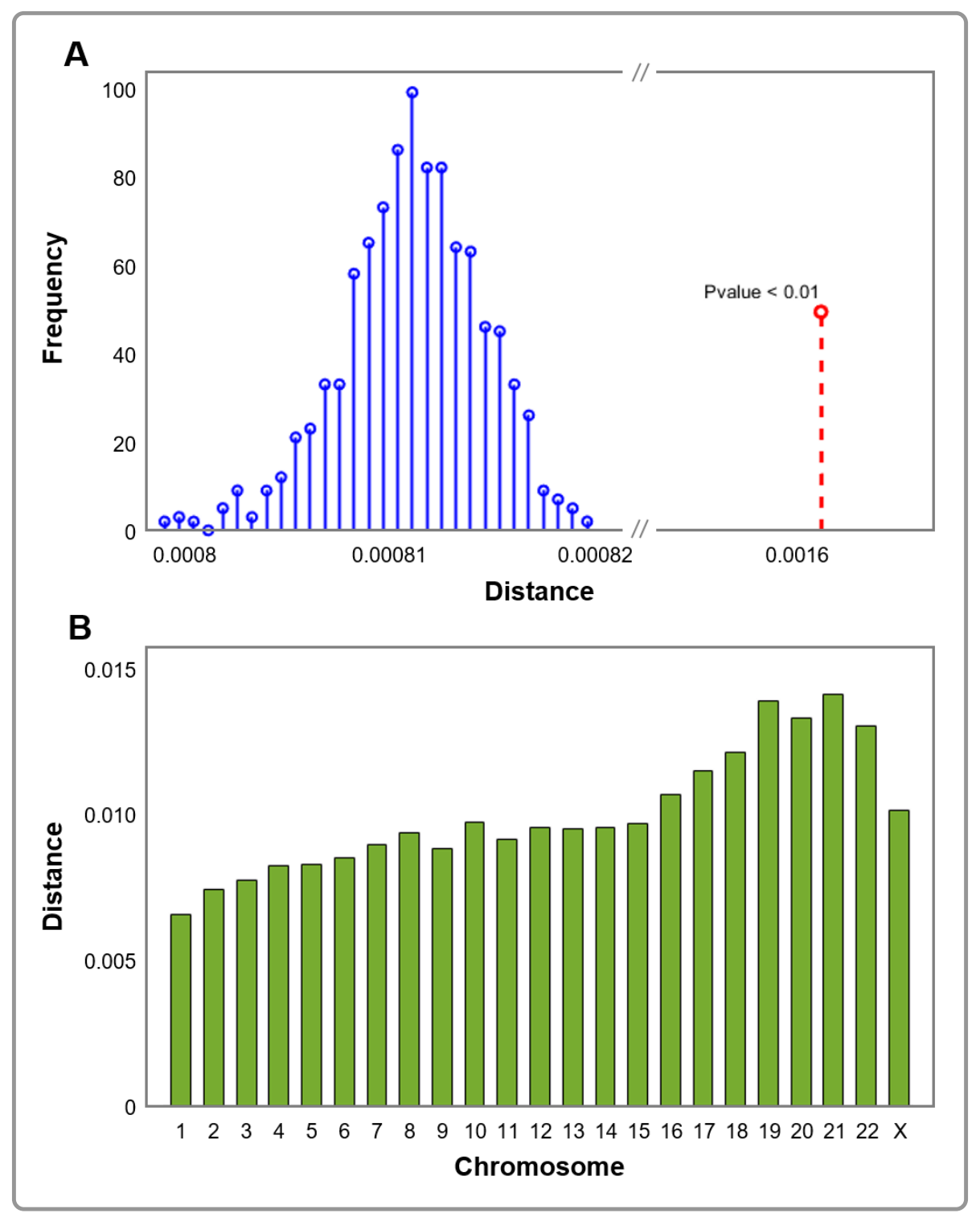

Figure S3: Hypergraph distance between two genome-wide hypergraphs derived from fibroblast and B lymphocytes. (A) Background distribution formed by measuring the hypergraph distances between the hypergraph derived from fibroblast and random hypergraphs. The actual distance between two genome-wide hypergraphs is also highlighted in red with a p-value $i$ 0.01. (B) Hypergraph distances between intra-chromosomal genomic hypergraphs between fibroblasts and B lymphocytes. 
bioRxiv preprint doi: https://doi.org/10.1101/2021.08.15.456363; this version posted September 2, 2021. The copyright holder for this preprint (which was not certified by peer review) is the author/funder. All rights reserved. No reuse allowed without permission.

\section{Supplementary Tables}

\begin{tabular}{|c|l|}
\hline File Type & \multicolumn{1}{c|}{ Description } \\
\hline .fastq & $\begin{array}{l}\text { Text file which contains unique identifiers for Pore-C reads and the raw sequences } \\
\text { contained within each read }\end{array}$ \\
\hline .pairs & $\begin{array}{l}\text { Text file which contains pairs of aligned genomic loci at base-pair resolution, which } \\
\text { can be grouped together using unique identifiers to construct multi-way contacts }\end{array}$ \\
\hline parquet & $\begin{array}{l}\text { Text file which contains aligned multi-way contacts at base-pair resolution, restric- } \\
\text { tion fragment assignments, and indicators for the quality of read alignment }\end{array}$ \\
\hline mcool & $\begin{array}{l}\text { Binary storage file which contains pairwise interactions from Pore-C data at mul- } \\
\text { tiple resolutions }\end{array}$ \\
\hline
\end{tabular}

Table S1: Descriptions of file types used within the computational framework (Figure 1).

\begin{tabular}{|c|c|c|c|c|c|c|}
\hline Order & $\begin{array}{c}\text { Multi-way } \\
\text { Contacts }\end{array}$ & $\begin{array}{c}\text { Transcription } \\
\text { Clusters }\end{array}$ & $\begin{array}{c}\text { Clusters with } \\
\mathbf{2} \text { 2 Genes }\end{array}$ & $\begin{array}{c}\text { Clusters with } \\
\mathbf{1} \text { Enhancer }\end{array}$ & $\begin{array}{c}\text { Clusters with } \\
\text { Common TFs }\end{array}$ & $\begin{array}{c}\text { Clusters with } \\
\text { Common MRs }\end{array}$ \\
\hline \multirow{2}{*}{3} & 379,165 & 11,261 & 2,986 & 2,914 & 715 & 336 \\
\cline { 2 - 7 } & 240,477 & 8,384 & 4,157 & 3,487 & 1,092 & 739 \\
\hline \multirow{2}{*}{4} & 181,554 & 3,254 & 1,214 & 1,208 & 80 & 34 \\
\cline { 2 - 7 } & 227,352 & 4,345 & 2,686 & 2,387 & 270 & 168 \\
\hline \multirow{2}{*}{5} & 98,272 & 1,021 & 473 & 467 & 8 & 6 \\
\cline { 2 - 7 } & 196,423 & 1,996 & 1,434 & 1,315 & 53 & 32 \\
\hline \multirow{2}{*}{$6+$} & 142,575 & 544 & 341 & 341 & 1 & 0 \\
\cline { 2 - 7 } & $1,000,231$ & 1,802 & 1,419 & 1,343 & 7 & 2 \\
\hline
\end{tabular}

Table S2: Summary of multi-way contacts with enhancers. Multi-way contacts from fibroblasts (gray rows, V1-V4) and B lymphocytes (white rows) are listed after different filtering criteria. Multi-way contacts are considered to be potential transcription clusters if all loci within the multi-way contact are accessible and at least one locus has binding from RNA Pol II. These multi-way contacts are then queried for nearby expressed genes. If a transcription cluster candidate has at least two expressed genes and at least one enhancer locus, we determine whether the genes have common transcription factors (TFs) through binding motifs. From the set of transcription clusters with common transcription factors, we calculate how many clusters are regulated by at least one master regulator (MR). 
bioRxiv preprint doi: https://doi.org/10.1101/2021.08.15.456363; this version posted September 2, 2021. The copyright holder for this preprint (which was not certified by peer review) is the author/funder. All rights reserved. No reuse allowed without permission.

\begin{tabular}{|c|c|c|c|c|c|c|c|c|c|c|}
\hline Chr & $\mathbf{1}$ & $\mathbf{2}$ & $\mathbf{3}$ & $\mathbf{4}$ & $\mathbf{5}$ & $\mathbf{6}$ & $\mathbf{7}$ & $\mathbf{8}$ & $\mathbf{9}$ & $\mathbf{1 0 +}$ \\
\hline 1 & 230 & 8,333 & 14,492 & 5,501 & 2,093 & 872 & 353 & 133 & 54 & 57 \\
\hline 2 & 241 & 9,183 & 15,548 & 6,180 & 2,373 & 873 & 437 & 197 & 71 & 95 \\
\hline 3 & 198 & 7,113 & 13,631 & 5,113 & 1,909 & 750 & 302 & 132 & 57 & 50 \\
\hline 4 & 190 & 6,342 & 11,147 & 4,360 & 1,642 & 598 & 284 & 113 & 56 & 28 \\
\hline 5 & 179 & 5,785 & 10,059 & 3,897 & 1,457 & 640 & 249 & 106 & 39 & 31 \\
\hline 6 & 167 & 5,361 & 9,368 & 3,551 & 1,364 & 511 & 207 & 75 & 39 & 27 \\
\hline 7 & 159 & 4,851 & 8,365 & 3,258 & 1,274 & 453 & 180 & 72 & 35 & 26 \\
\hline 8 & 143 & 4,321 & 7,596 & 2,864 & 1,069 & 393 & 154 & 71 & 32 & 16 \\
\hline 9 & 122 & 2,722 & 4,899 & 1,831 & 614 & 209 & 76 & 31 & 8 & 6 \\
\hline 10 & 134 & 4,010 & 7,185 & 2,695 & 912 & 337 & 144 & 63 & 23 & 14 \\
\hline 11 & 133 & 3,698 & 6,613 & 2,533 & 890 & 330 & 101 & 62 & 14 & 14 \\
\hline 12 & 132 & 3,815 & 6,630 & 2,497 & 878 & 324 & 114 & 47 & 17 & 12 \\
\hline 13 & 97 & 2,627 & 4,767 & 1,751 & 588 & 247 & 82 & 37 & 11 & 8 \\
\hline 14 & 87 & 2,209 & 3,932 & 1,366 & 509 & 140 & 51 & 19 & 10 & 2 \\
\hline 15 & 82 & 1,623 & 2,812 & 991 & 298 & 98 & 29 & 16 & 2 & 1 \\
\hline 16 & 80 & 1,650 & 3,126 & 1,181 & 363 & 127 & 39 & 11 & 5 & 2 \\
\hline 17 & 82 & 1,345 & 2,374 & 732 & 201 & 66 & 20 & 4 & 3 & 2 \\
\hline 18 & 77 & 1,828 & 3,230 & 1,128 & 368 & 149 & 51 & 16 & 10 & 4 \\
\hline 19 & 56 & 873 & 1,455 & 462 & 118 & 41 & 7 & 3 & 0 & 0 \\
\hline 20 & 64 & 1,247 & 2,238 & 783 & 222 & 69 & 27 & 6 & 3 & 0 \\
\hline 21 & 39 & 453 & 809 & 264 & 79 & 18 & 6 & 1 & 0 & 0 \\
\hline 22 & 39 & 438 & 728 & 206 & 43 & 13 & 3 & 0 & 1 & 0 \\
\hline $\mathrm{X}$ & 151 & 1,078 & 1,852 & 882 & 350 & 119 & 43 & 17 & 10 & 0 \\
\hline $\mathrm{Y}$ & 23 & 70 & 93 & 25 & 6 & 4 & 0 & 0 & 0 & 0 \\
\hline
\end{tabular}

Table S3: Fibroblast intra-chromosome contact orders (1 Mb resolution).

\begin{tabular}{|c|c|c|c|c|c|c|c|c|c|c|}
\hline Chr & $\mathbf{1}$ & $\mathbf{2}$ & $\mathbf{3}$ & $\mathbf{4}$ & $\mathbf{5}$ & $\mathbf{6}$ & $\mathbf{7}$ & $\mathbf{8}$ & $\mathbf{9}$ & $\mathbf{1 0 +}$ \\
\hline 1 & 232 & 4,725 & 17,254 & 16,396 & 10,752 & 6,836 & 4,248 & 2,663 & 1,652 & 3,130 \\
\hline 2 & 243 & 4,964 & 16,695 & 16,702 & 11,787 & 7,891 & 5,172 & 3,432 & 2,288 & 4,631 \\
\hline 3 & 199 & 4,055 & 16,351 & 14,795 & 9,846 & 6,376 & 4,023 & 2,691 & 1,759 & 3,293 \\
\hline 4 & 191 & 3,725 & 13,399 & 13,309 & 9,192 & 6,218 & 4,081 & 2,632 & 1,698 & 3,301 \\
\hline 5 & 180 & 3,449 & 12,352 & 12,458 & 8,799 & 5,810 & 3,672 & 2,358 & 1,548 & 2,728 \\
\hline 6 & 171 & 3,300 & 11,249 & 11,640 & 7,862 & 5,307 & 3,235 & 2,139 & 1,376 & 2,486 \\
\hline 7 & 160 & 3,086 & 11,350 & 11,272 & 7,758 & 5,101 & 3,223 & 2,213 & 1,391 & 2,613 \\
\hline 8 & 145 & 2,895 & 10,755 & 10,608 & 7,333 & 4,778 & 3,141 & 2,068 & 1,289 & 2,477 \\
\hline 9 & 124 & 2,399 & 9,360 & 8,535 & 5,847 & 3,802 & 2,393 & 1,572 & 931 & 1,753 \\
\hline 10 & 134 & 2,580 & 9,429 & 9,273 & 6,203 & 4,113 & 2,576 & 1,632 & 1,020 & 1,856 \\
\hline 11 & 134 & 2,505 & 8,979 & 8,661 & 5,795 & 3,629 & 2,250 & 1,408 & 852 & 1,530 \\
\hline 12 & 134 & 2,376 & 8,329 & 8,200 & 5,465 & 3,449 & 2,192 & 1,344 & 833 & 1,304 \\
\hline 13 & 99 & 1,763 & 6,665 & 6,527 & 4,585 & 2,935 & 1,834 & 1,078 & 635 & 1,159 \\
\hline 14 & 89 & 1,531 & 5,909 & 5,884 & 3,863 & 2,236 & 1,430 & 858 & 514 & 843 \\
\hline 15 & 85 & 1,349 & 5,064 & 5,130 & 3,354 & 1,964 & 1,164 & 706 & 372 & 582 \\
\hline 16 & 83 & 1,444 & 5,993 & 5,722 & 3,769 & 2,437 & 1,419 & 808 & 494 & 813 \\
\hline 17 & 84 & 1,355 & 4,278 & 4,294 & 2,612 & 1,514 & 882 & 475 & 271 & 366 \\
\hline 18 & 80 & 1,478 & 6,015 & 5,569 & 3,616 & 2,359 & 1,380 & 861 & 549 & 840 \\
\hline 19 & 57 & 884 & 3,335 & 3,251 & 1,998 & 1,141 & 664 & 383 & 224 & 252 \\
\hline 20 & 65 & 1,053 & 4,344 & 4,149 & 2,684 & 1,656 & 902 & 547 & 315 & 441 \\
\hline 21 & 40 & 481 & 2,285 & 2,245 & 1,392 & 747 & 402 & 235 & 96 & 127 \\
\hline 22 & 39 & 445 & 1,739 & 1,795 & 1,098 & 512 & 255 & 127 & 59 & 72 \\
\hline $\mathrm{X}$ & 156 & 3,681 & 12,552 & 11,686 & 8,206 & 5,915 & 3,941 & 2,738 & 1,950 & 4,414 \\
\hline
\end{tabular}

Table S4: GM12878 intra-chromosome contact orders (1 Mb resolution). 